\title{
Linking applicatory functions to the 3-position of pyrrole by click chemistry
}

\author{
Sebastian Karsten, ${ }^{a}$ Alexandrina Nan, ${ }^{b}$ Jürgen Liebscher ${ }^{a, b^{*}}$ \\ ${ }^{a}$ Institute of Chemistry, Humboldt-University Berlin, Brook-Taylor-Str. 2, 12489 Berlin, \\ Germany \\ ${ }^{b}$ National Institute of Research and Development for Isotopic and Molecular Technologies \\ (INCDTIM), Str. 65-103 Donath, Cluj-Napoca, Cluj, RO-400293, Romania \\ E-mail: liebscher@chemie.hu-berlin.de
}

\begin{abstract}
A straight forward and reliable method was developed to tether recognition functions to a side chain in position 3 of pyrroles via triazole linkage. The products are precursors for functionalized polypyrroles, e. g. for coating magnetic nanoparticles or selective electrodes. A pyrrole with an azido function located at the terminus of a side chain in position 3 was submitted to coppercatalyzed Meldal-Sharpless click reaction with alkynes bearing biotin, nitrilotriacetic acid or a RGD-containing cyclopentapeptide. The latter presents a very versatile building block for the introduction of the RGD-moiety in a variety of potential substrates.
\end{abstract}

Keywords: Pyrroles, alkynes, azides, cycloadditions, biomolecules

\section{Introduction}

Polypyrrole (PPy) is a conducting polymer, ${ }^{1}$ which has found wide applications, such as in membranes $^{2}$ and in coating of electrodes ${ }^{3-5}$ or of nanoparticles. ${ }^{6-12}$ Amongst nanoparticles magnetic nanoparticles have attracted wide interest in nanotechnology and nanomedicine. ${ }^{13,14}$ The scope of polypyrroles can be considerably extended when functions, such as reactive groups, biological recognition functions, ligands for metal complexation, or catalytic units are introduced. ${ }^{15,16}$ As attachment points positions 1 and 3 of the pyrrole ring are preferred, because oxidative polymerizations of pyrroles to PPy run preferably via positions 2 and 5. In most cases, the functions are not directly attached to the pyrrole ring but are connected via linkers. This provides more freedom to the attached functions ${ }^{17}$ and the effect of the substituents on the electron density in the pyrrole ring can be controlled by the type of linker. From the practical point of view it is advisable to introduce the function at a late stage of the synthetic sequence, $i$. e. to obtain a precursor which can accept the respective function in the last step. Following this strategy, we recently developed pyrroles with azido or propargyl groups linked to position 1 of 
the pyrrole ring. ${ }^{9}$ These derivatives can easily undergo Cu-catalyzed Meldal-Sharpless click reaction ${ }^{18-20}$ with functions (glucose, cholesterol, biotin, nucleosides) equipped with an alkyne or azido moiety, respectively, resulting in 1,2,3-triazole linkages. A major advantage of this clickcoupling method is the fact that many functional groups are tolerated thus avoiding protective group strategies. Since substituents in position 1 of the pyrrole generally somewhat hamper the oxidative polymerization to polypyrroles ${ }^{21}$ and can give rise to branching ${ }^{22}$ we seek to develop a way that allows to link interesting functions to position 3 of the pyrrole ring, thus enabling easier oxidative polymerization to polypyrroles. In this context it is worth mentioning that a DNAsequence was linked to position 3 of a polypyrrole film by amide formation. ${ }^{23}$

\section{Results and Discussion}

The 3-substituted azido-containing 1 fulfills all preconditions for the envisaged strategy. It carries a terminal azido group which is connected to position 3 via a polar oligo-ethylene glycol butyramide linker and does not decrease the electron density in the pyrrole ring. Thus it is a good candidate for both the $\mathrm{Cu}$-catalyzed click reaction with alkynes and the subsequent oxidative polymerization to polypyrroles. As suitable coupling partners for the azido-functionalized pyrrole 1 we chose alkynes equipped with biotin (recognition of avidin or streptavidin), ${ }^{24}$ nitrilotriacetic acid (complexing metal ions) and a RGD-containing cyclopentapeptide (medical application, vide infra) as interesting applicatory functions. The respective biotin-substituted alkyne $\mathbf{2 a}$ is available by reaction of biotinylsuccinate ${ }^{25}$ with

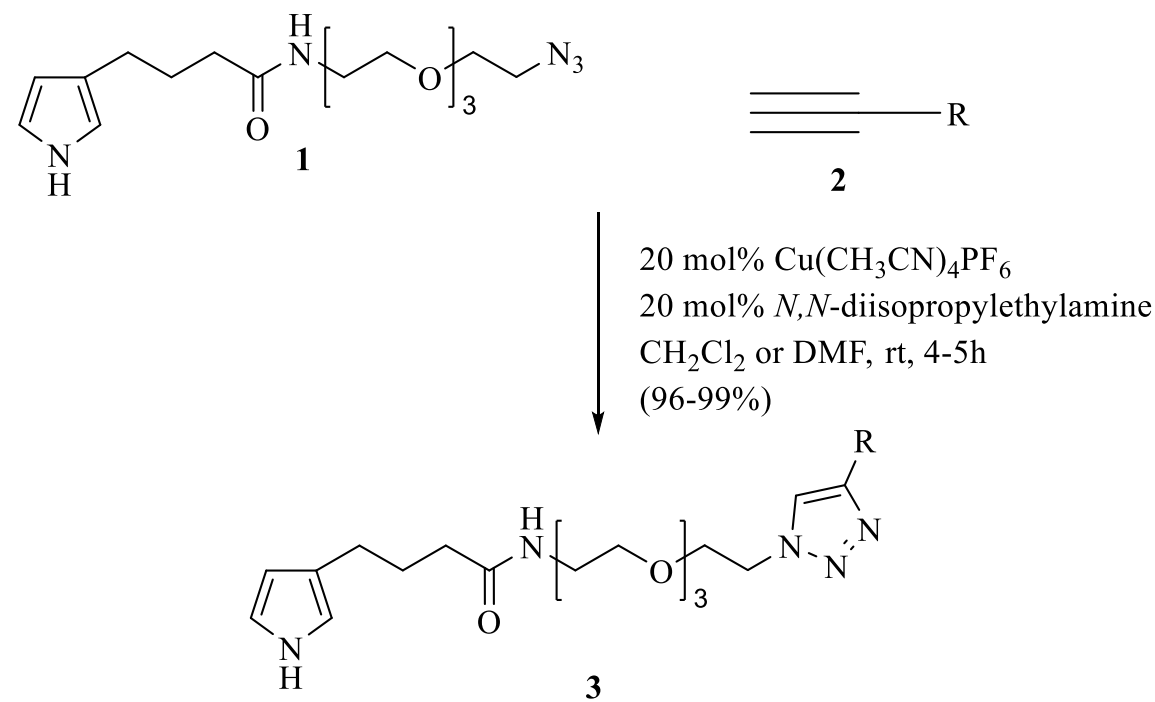

Scheme 1. Synthesis of functionalized pyrroles 3 by $\mathrm{Cu}$-catalyzed click reaction of azidopyrrole $\mathbf{1}$ with alkynes $\mathbf{2}$. 
aminoethyltriethylenglycol propargylether in a straight forward one step reaction. The two unknown nitrilotriacetic acid derivatives $\mathbf{2 b}$ and $\mathbf{2 c}$ were obtained from the lysine derivative $\mathbf{5}^{26}$ by 1-ethyl-3-(3-dimethylaminopropyl)carbodiimide (EDC) assisted amide formation (Scheme 2).

Table 1.1,2,3-Triazoles 3 by Meldal-Sharpless click reaction

3 Product 3 3d




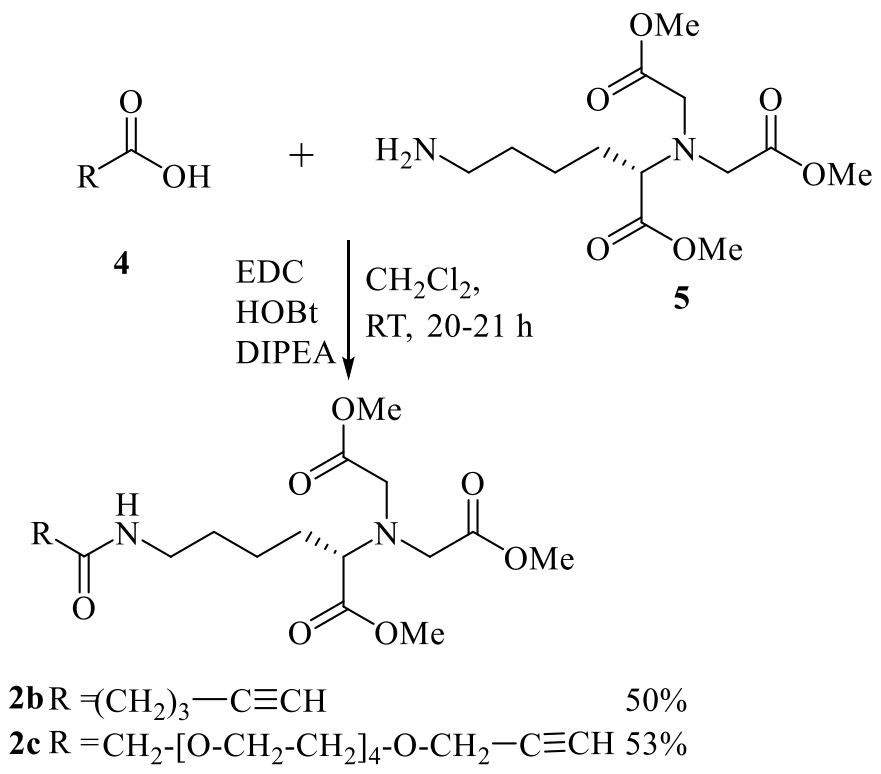

Scheme 2. Synthesis of alkyne-functionalized nitrilotriacetic esters $\mathbf{2 b}$ and $\mathbf{2 c}$.

In order to obtain a suitable RGD-containing alkyne we designed a cyclo[Arg-Gly-Asp-DPhe-Glu] wherein the properties of the RGD-sequence are not likely to be affected by the alkyne functionalization. Cyclic RGD-containing pentapeptides were applied as selective antagonists for the $\alpha_{\mathrm{v}} \beta_{3}$ integrin receptor and have found wide applications. ${ }^{27}$ For covalent linking of such cyclopeptides, modified or unmodified $\varepsilon$-amino lysine side chains were often used. ${ }^{28-32}$ Recently $\mathrm{Cu}$-catalyzed click reaction was performed at such a RGD-containing cyclopeptide wherein prior the lysine amino group was transferred into an azido group. ${ }^{30}$ In another way the lysine amino group was acylated by propiolic acid and used to introduce an RGD-cyclopeptide into xylose by click chemistry. ${ }^{32}$ Here, we chose the side chain of a glutamate in a cyclo[Arg-Gly-Asp-D-PheGlu] peptide as attachment point for an alkyne moiety (Scheme 3). The synthesis of the respective propargylamide 2d started with Fmoc-protected glycine-loaded chlorotrityl resin 6. The pentapeptide 7 was built up by solid phase peptide synthesis wherein Pbf-protected Arg was used in the first and $N$-propargylated Glu in the second coupling step. The pentapeptide 7 was obtained in $77 \%$ after chromatographic purification. Macrolactamization was implemented by benzotriazol-1-yl-oxytripyrrolidinophosphonium hexafluorophosphate (PyBOP) activation providing high yield (93\%) of the cyclopentapeptide $\mathbf{8}$ which was treated with trifluoroacetic acid (TFA) giving rise to the deprotected product $\mathbf{2 d}$. This cyclopentapeptide $\mathbf{2 d}$ was obtained in scales of hundred of $\mathrm{mg}$. It represents a novel very versatile RGD-derivative which is an excellent candidate for $\mathrm{Cu}$-catalyzed Meldal-Sharpless click reactions with a variety of azidofunctionalized substrates. 


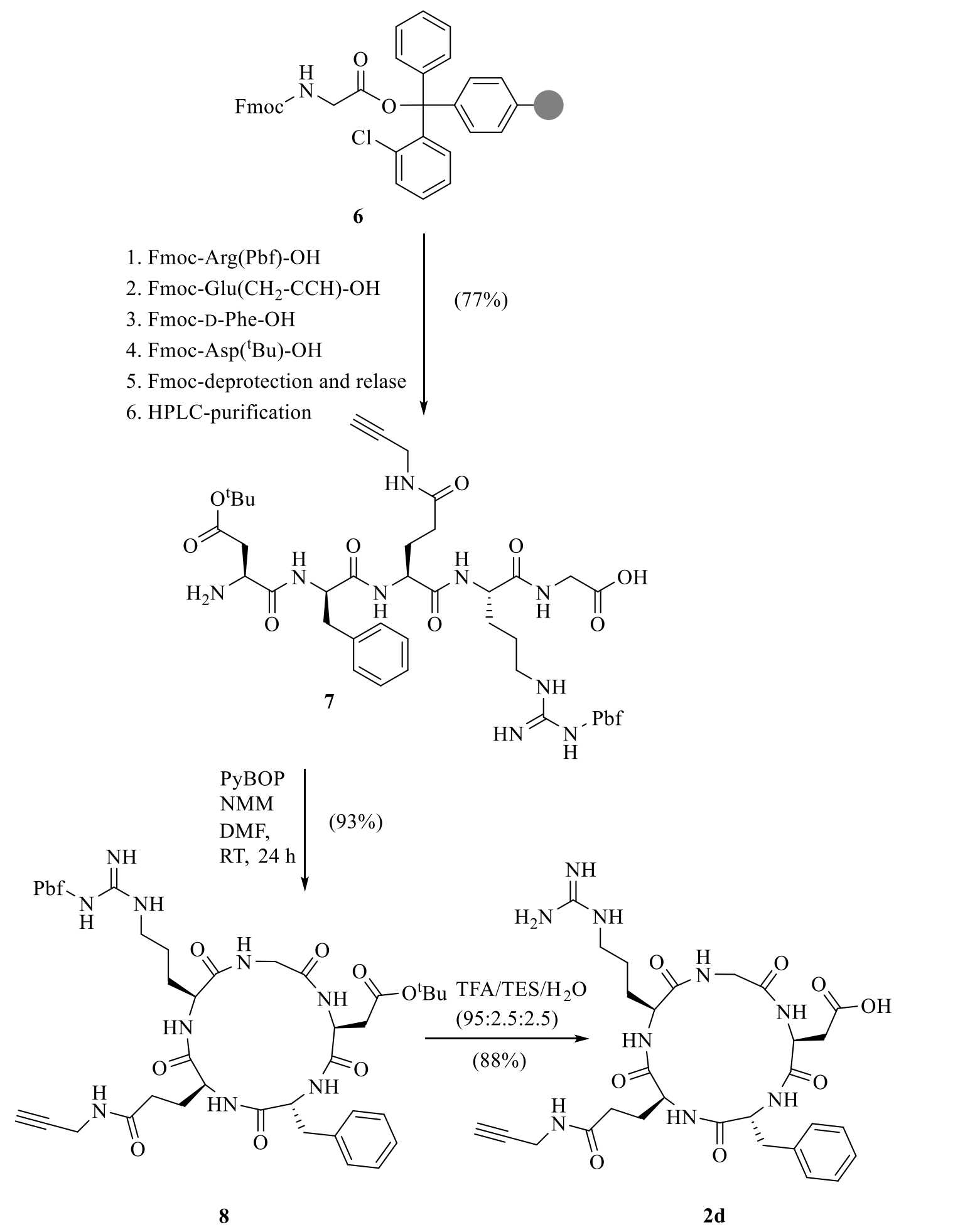

Scheme 3. Synthesis of propargyl-functionalized RGD-containing cyclopentapeptide 2d.

With all the starting materials $\mathbf{1}$ and $\mathbf{2}$ in hand we performed $\mathrm{Cu}$-catalyzed Meldal-Sharpless click reaction using $\mathrm{Cu}\left(\mathrm{CH}_{3} \mathrm{CN}\right)_{4} \mathrm{PF}_{6}$ as catalyst. After the reaction, the copper catalyst was removed from the reaction mixture by Cuprisorb ${ }^{\mathrm{TM}}$ rendering the work up very easy. Pleasingly, 
all products 3 were obtained in excellent yields (> 95\%) (Scheme 1, Table 1). They were characterized by spectroscopic methods and are presently investigated in our laboratories for further application in the preparation of functionalized core-shell nanoparticles.

As a proof of principle for application of the triazolyl-substituted pyrroles $\mathbf{3}$ as components for the synthesis of functionalized polypyrroles, we submitted the biotin derivative 3a to oxidative copolymerization (ammonium persulfate as oxidizing reagent) with unsubstituted pyrrole in the presence of magnetite nanoparticles 9 stabilized by a double layer of lauric acid (Scheme 4). The resulting polypyrrole-magnetite core shell nanoparticles $\mathbf{1 0}$ can easily isolated by magnetic separation with an external magnet and decantation leaving back polypyrrole which is not linked to the nanoparticles in the mother liquor. The incorporation of biotin moieties in the magnetic polypyrrole-magnetite core shell nanoparticles $\mathbf{1 0}$ is proved by the appearance of typical bands in the FTIR-spectrum (Figure 1). For comparison the FTIR-spectrum of the precursor magnetite nanoparticles 9 covered by a double layer of lauric acid is shown too (Fig. 1). The bands located at $616 \mathrm{~cm}^{-1}$ found in both spectra is specific for $\mathrm{Fe}-\mathrm{O}$ bond in magnetite. The spectrum of 10 shows a band at $1642 \mathrm{~cm}^{-1}$ typical for the carbonyl group of the ureido moiety of biotin. A very broad intensive band situated at $3233 \mathrm{~cm}^{-1}$ can be assigned to $\mathrm{NH}$ stretching vibrations of the $\mathrm{NH}$ groups of biotin and to the $\mathrm{CH}$ stretching bands of the pyrrole rings. The adsorption band situated at $1547 \mathrm{~cm}^{-1}$ is ascribed to the collective vibration mode of intra-ring and inter-ring $\mathrm{C}=\mathrm{C} / \mathrm{C}-\mathrm{C}$ of polypyrrole chains. At $1433 \mathrm{~cm}^{-1}$ appears the adsorption band specific for $\mathrm{C}-\mathrm{N}$ bonds.

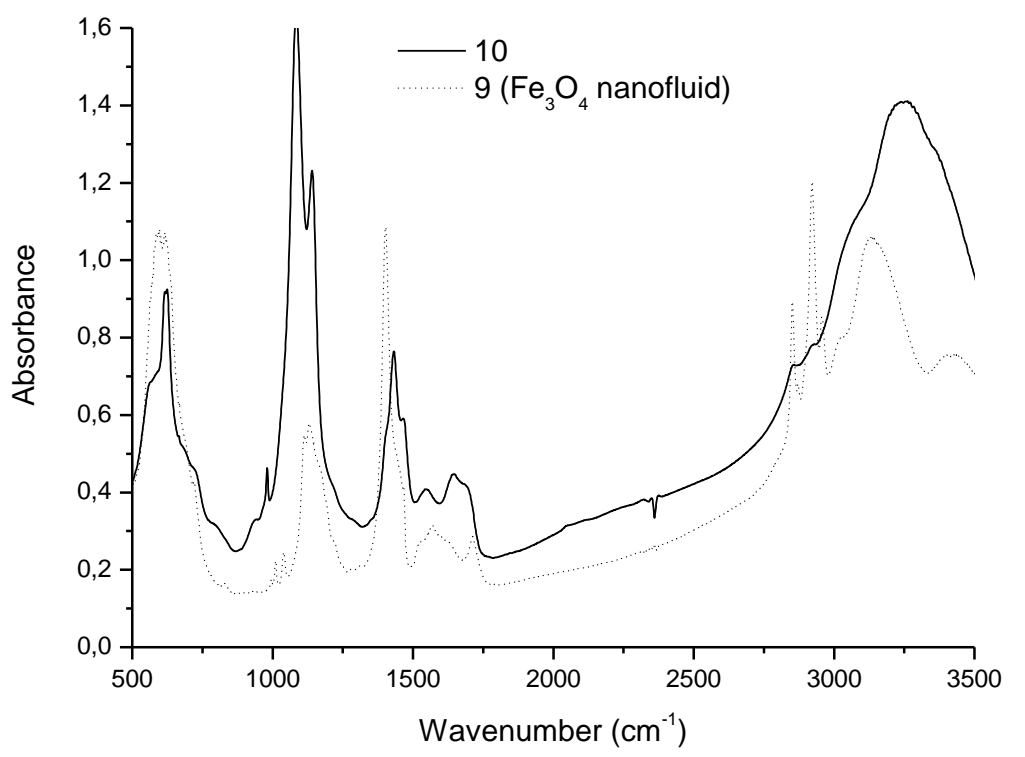

Figure 1. FTIR-Spectra (in KBr) of biotin-functionalized polypyrrole-magnetite nanoparticles $\mathbf{1 0}$ and the precursor magnetite nanoparticles $\mathbf{9}$ stabilized by a double layer of lauric acid. 


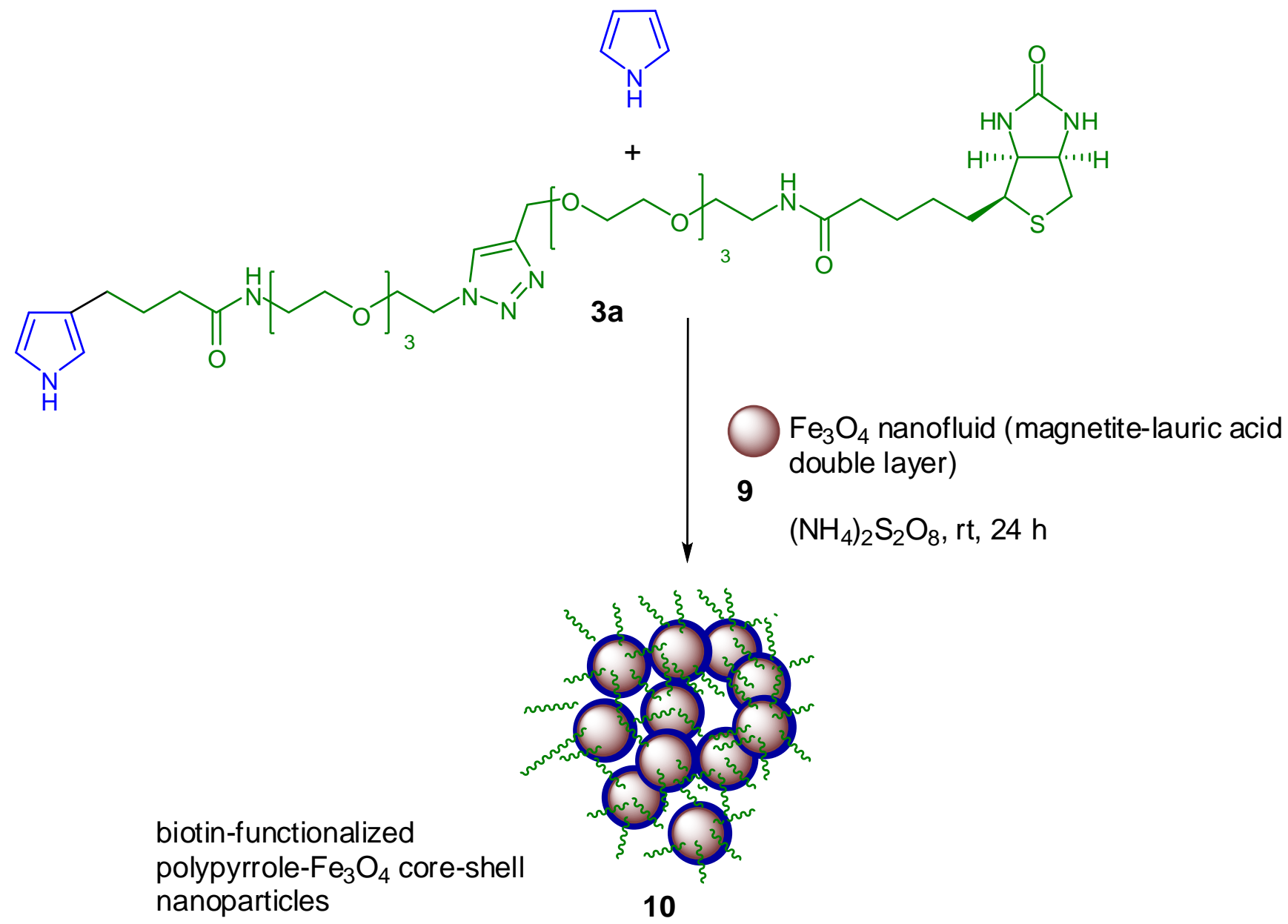

$\mathrm{Fe}_{3} \mathrm{O}_{4}$ iron oxide nanoparticles stabilzed by a double layer of oleic acid

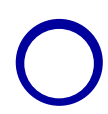

polypyrrole shell covering the magnetite core

m

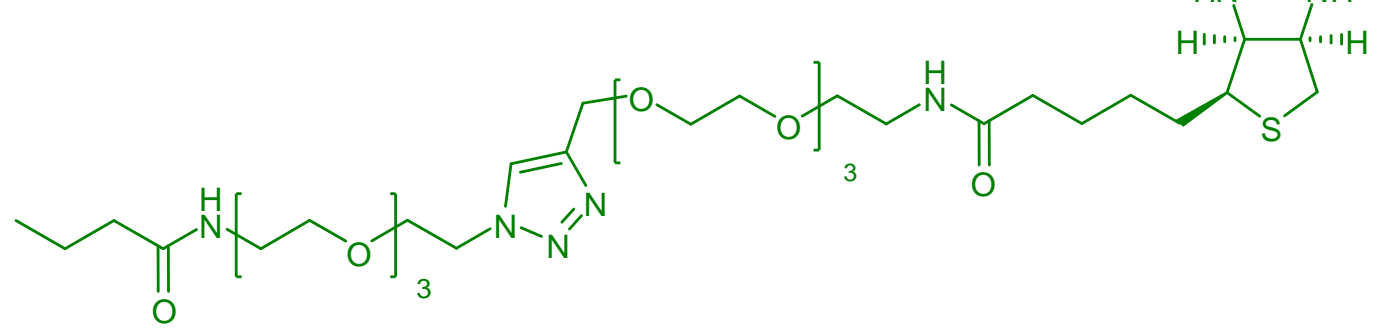

Scheme 4. Synthesis of biotin-functionalized polypyrrole-magnetite core-shell nanoparticles $\mathbf{1 0}$. 


\section{Conclusions}

In summary, novel pyrroles were developed which contain interesting applicatory functions (biotin, nitrilotriacetic acid, RGD-containing cyclopentapeptide) tethered to a substituent in position 3 via 1,2,3-triazole linkage. These pyrroles represent promising precursor for functionalized polypyrroles useful for magnetic core-shell nanoparticles or as electrode coatings. The pyrroles were obtained in a straight forward way by $\mathrm{Cu}$-catalyzed Meldal-Sharpless click reaction of a pyrrole with an azido group tethered to position 3 via a linker and alkyne moieties equipped with the respective functions. Amongst the latter the alkyne -containing RGDcyclopentapeptide represents a versatile building block for potential tethering the RGD-sequence to various targets. The biotin-triazole-pyrrole conjugate was applied in the preparation of new magnetic polypyrrole-magnetite core-shell nanoparticles equipped with biotin as recognition function for avidin or streptavidin.

\section{Experimental Section}

General. Chemicals were purchased from Aldrich and Acros. Silica gel $60(0.04-0.063 \mathrm{~mm}$, Acros) was used for preparative column chromatography. Melting points were determined on a Boetius hotstage apparatus and were uncorrected. ${ }^{1} \mathrm{H}$ NMR and ${ }^{13} \mathrm{C}$ NMR spectra were recorded at 500 or 300 and 125 or $75 \mathrm{MHz}$, respectively, on a Bruker AV-500 or Bruker AV-300 with TMS as an internal standard. High resolution mass spectra (ESI) were measured with a Thermo Finnigan LTQ-FT-ICR-MS with $\mathrm{MeOH}$ as a solvent. FTIR spectra were measured with a Jasco FT/IR-4200-Spectrometer.

1,2,3-Triazoles 3. General procedure. A solution of azidopyrrole $\mathbf{1}^{33}(200 \mu \mathrm{mol})$ and the alkyne $2(200 \mu \mathrm{mol})$ in $\mathrm{CH}_{2} \mathrm{Cl}_{2}(10 \mathrm{ml})$ was degassed by argon. $\mathrm{Cu}\left(\mathrm{CH}_{3} \mathrm{CN}\right)_{4} \mathrm{PF}_{6}(15 \mathrm{mg}, 40 . \mu \mathrm{mol})$ and $N$-ethyldiisopropylamine (DIPEA) $(7 \mu \mathrm{l}, 40 . \mu \mathrm{mol})$ were added and the mixture was stirred at $\mathrm{rt}$ until the reaction went to completion (TLC, about 4-5 h). Cuprisorb ${ }^{\mathrm{TM}}$ was added and stirring was continued for $60 \mathrm{~min}$. After filtration the filtrate was concentrated under vacuum by a rotary evaporator leaving behind the product.

4-[(2-[2-(2-[2-(Biotinylamino)ethoxy]ethoxy)ethoxy]ethoxy)methyl]-1-[(2-[2-(2-[4-(1Hpyrrol-3-yl)-butanoylamino]ethoxy)ethoxy]ethoxy)ethyl]-1H-1,2,3-triazole (3a). Reaction $\begin{array}{llllllllll}\text { time } 5 \mathrm{~h} \text {. Highly viscous dark brown oil; yield: } 156 \mathrm{mg} & (96 \%), & \mathrm{R}_{\mathrm{f}} & 0.14\end{array}$ $\left(\mathrm{CH}_{2} \mathrm{Cl}_{2} / \mathrm{MeOH} / \mathrm{HCOOH}, 90: 10: 1\right) .{ }^{1} \mathrm{H}-\mathrm{NMR}\left(\mathrm{CDCl}_{3}, 500 \mathrm{MHz}\right): \delta 1.38\left(\mathrm{~m}, 2 \mathrm{H}, \mathrm{CH}_{2}-\mathrm{C}_{2}-\right.$ $\mathrm{CH}_{2}$ ), 1.63 (m, 4H, CH biotin- $\left.\underline{\mathrm{C}}_{2}-\mathrm{C}_{2}\right), 1.85$ (m, 2H, $\left.\mathrm{CH}_{2}-\mathrm{C}_{2}-\mathrm{CH}_{2}\right), 2.16\left(\mathrm{t}, 4 \mathrm{H}, J\right.$ 7.4, $\mathrm{C}_{\mathrm{ar}}-\mathrm{CH}_{2}-$ $\mathrm{CH}_{2}, \mathrm{CH}_{2}-\underline{\mathrm{C}}_{2}-\mathrm{CO}$ ), 2.47 (t, $2 \mathrm{H}, J$ 7.4, $\mathrm{CH}_{2}-\underline{\mathrm{C}}_{2}-\mathrm{CO}$ ), 2.68 (d, 1H, J 12.7, $\mathrm{CH}_{\text {biotin }}-\underline{\mathrm{C}}_{2}-\mathrm{S}$ ), 2.84 (dd, $1 \mathrm{H}, J_{1} 4.8, J_{2}$ 12.7, $\mathrm{CH}_{\text {biotin- }} \underline{\mathrm{C}}_{2}-\mathrm{S}$ ), 3.08 (m, 1H, $\underline{\mathrm{C}}_{\text {biotin- }} \mathrm{CH}_{2}-\mathrm{CH}_{2}$ ), 3.37 (m, 4H, 2xNH$\left.\mathrm{C}_{2}-\mathrm{CH}_{2}-\mathrm{O}\right), 3.50\left(\mathrm{~m}, 4 \mathrm{H}, 2 \times \mathrm{xNH}-\mathrm{CH}_{2}-\underline{\mathrm{C}}_{2}-\mathrm{O}\right), 3.54-3.66\left(\mathrm{~m}, 20 \mathrm{H}, 5 \mathrm{xO}-\mathrm{C}_{2}-\underline{\mathrm{C}}_{2}-\mathrm{O}\right), 3.81(\mathrm{t}$, $2 \mathrm{H}, J$ 5.1, $\left.\underline{\mathrm{C}}_{2}-\mathrm{CH}_{2}-\mathrm{N}_{\text {triazole }}\right), 4.25$ (dd, $1 \mathrm{H}, J_{1} 4.5, J_{2} 7.1, \mathrm{NH}_{\text {biotin- }} \underline{\mathrm{CH}}-\mathrm{CH}-\mathrm{S}$ ), 4.44 (dd, $1 \mathrm{H}, J_{1}$ 
5.1, $J_{2}$ 7.1, $\mathrm{NH}_{\text {biotin- }} \underline{\mathrm{C}} \mathrm{H}-\mathrm{CH}_{2}-\mathrm{S}$ ), 4.47 (t, $2 \mathrm{H}, J$ 5.1, $\mathrm{CH}_{2}-\mathrm{C}_{2}-\mathrm{N}_{\text {triazole }}$ ), 4.62 (s, 2H, O-C $\underline{\mathrm{H}}_{2}-$

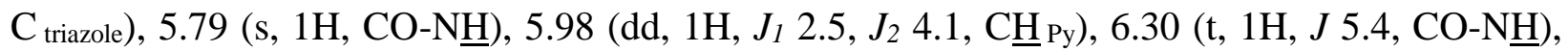
$6.48(\mathrm{~s}, 1 \mathrm{H}, \mathrm{CO}-\mathrm{N} \underline{\mathrm{H}}), 6.52\left(\mathrm{~m}, 1 \mathrm{H}, \mathrm{C}_{\mathrm{Py}}\right), 6.64\left(\mathrm{dd}, 1 \mathrm{H}, J_{1} 2.5, J_{2} 4.7, \underline{\mathrm{C}}_{\mathrm{Py}}\right), 6.83(\mathrm{t}, 1 \mathrm{H}, J 5.5$, $\mathrm{CO}-\mathrm{N} \underline{\mathrm{H}}), 7.69$ (s, $\left.1 \mathrm{H}, \mathrm{C}_{\text {triazole }}\right), 8.84$ (s, br, $\left.1 \mathrm{H}, \mathrm{NH}_{\mathrm{Py}}\right) .{ }^{13} \mathrm{C}-\mathrm{NMR}\left(\mathrm{CDCl}_{3}, 125 \mathrm{MHz}\right): \delta 25.6$ $\left(\mathrm{CH}_{2}-\mathrm{CH}_{2}-\mathrm{CH}_{2}\right), 26.4\left(\mathrm{CH}_{2}-\underline{C H}_{2}-\mathrm{CH}_{2}\right), 27.1\left(\mathrm{CH}_{2}-\underline{\mathrm{CH}}_{2}-\mathrm{CO}\right), 28.1\left(\mathrm{CH}_{\text {biotin- }}-\mathrm{CH}_{2}-\underline{\mathrm{CH}}_{2}\right), 28.3$ $\left(\mathrm{CH}_{\text {biotin- }}-\mathrm{CH}_{2}-\mathrm{CH}_{2}\right), 35.9\left(\mathrm{CH}_{2}-\mathrm{CH}_{2}-\mathrm{CO}\right), 36.1\left(\mathrm{C}_{\mathrm{ar}}-\mathrm{CH}_{2}-\mathrm{CH}_{2}\right), 39.1\left(\mathrm{NH}-\mathrm{CH}_{2}-\mathrm{CH}_{2}-\mathrm{O}\right), 39.2$ $\left(\mathrm{NH}-\underline{\mathrm{CH}}_{2}-\mathrm{CH}_{2}-\mathrm{O}\right), 40.5\left(\mathrm{CH}_{\text {biotin }} \underline{\mathrm{C}} \mathrm{H}_{2}-\mathrm{S}\right), 50.2\left(\mathrm{CH}_{2}-\underline{\mathrm{CH}}_{2}-\mathrm{N}_{\text {triazole }}\right), 55.7\left(\underline{\mathrm{C}} \mathrm{H}_{\text {biotin }}-\mathrm{CH}_{2}-\mathrm{CH}_{2}\right)$, $60.3\left(\mathrm{NH}_{\text {biotin- }} \underline{\mathrm{CH}}-\mathrm{CH}_{2}-\mathrm{S}\right), 61.8$ ( $\left.\mathrm{NH}_{\text {biotin- }} \underline{\mathrm{CH}}-\mathrm{CH}-\mathrm{S}\right), 64.5\left(\mathrm{O}-\underline{\mathrm{CH}}_{2}-\mathrm{C}_{\text {triazole }}\right), 69.4\left(\mathrm{O}-\underline{\mathrm{CH}}_{2}-\mathrm{CH}_{2}-\right.$ $\mathrm{O}), 69.6\left(\mathrm{O}-\mathrm{CH}_{2}-\mathrm{CH}_{2}-\mathrm{O}\right), 69.9\left(2 \times \mathrm{O}-\underline{\mathrm{CH}}_{2}-\mathrm{CH}_{2}-\mathrm{O}\right), 70.1\left(\mathrm{O}-\mathrm{CH}_{2}-\mathrm{CH}_{2}-\mathrm{O}\right), 70.2\left(\mathrm{O}-\mathrm{CH}_{2}-\mathrm{CH}_{2}-\mathrm{O}\right)$, $70.4\left(2 \times{ }^{x}-\underline{C H}_{2}-\mathrm{CH}_{2}-\mathrm{O}\right), 70.5\left(5 \mathrm{xO}-\underline{\mathrm{CH}}_{2}-\mathrm{CH}_{2}-\mathrm{O}\right), 108.2\left(\underline{\mathrm{CH}} \mathrm{Py}_{\mathrm{Py}}\right), 115.3\left(\underline{\mathrm{C}} \mathrm{H}_{\mathrm{Py}}\right), 117.8\left(\underline{\mathrm{CH}} \mathrm{Py}_{\mathrm{Py}}\right)$, $122.9\left(\underline{\mathrm{C}}_{\mathrm{q}}, \mathrm{Py}\right), 124.0\left(\underline{\mathrm{CH}}_{\text {triazole }}\right), 144.7\left(\underline{\mathrm{C}}_{\mathrm{q}, \text { triazole }}\right), 164.1(\underline{\mathrm{C}}=\mathrm{O}), 173.5(2 \mathrm{x} \underline{\mathrm{C}}=\mathrm{O})$.

FTIR $\left(\mathrm{cm}^{-1}\right): 3294 v(\mathrm{NH} \&$ triazole $), 2924 v_{\text {as }}\left(\mathrm{CH}_{2}\right), 2859 v_{\mathrm{s}}\left(\mathrm{CH}_{2}\right), 1698 v(\mathrm{C}=\mathrm{O}$ biotin $), 1648$ $v(\mathrm{C}=\mathrm{O}), 1550 \quad v\left(\mathrm{C}=\mathrm{C}_{\text {triazole }}\right), \quad 1455 \delta\left(\mathrm{CH}_{2}\right), 1093 v_{\text {as }}\left(\mathrm{C}-\mathrm{O}-\mathrm{C}_{\mathrm{TEG}}\right), 843 \gamma\left(\mathrm{CH}_{\text {triazole }}\right), 730$ $\delta\left(\mathrm{CH}_{\mathrm{Py}} \text { ). HRMS (ESI): } \mathrm{m} / z \text { [M+H] }\right]^{+}$calcd for $\mathrm{C}_{37} \mathrm{H}_{63} \mathrm{~N}_{8} \mathrm{O}_{10} \mathrm{~S}: 811.4382$; found: 811.4384 .

$N_{\alpha}, N_{\alpha}$-Bis(2-methoxycarbonylmethyl)- $N_{\varepsilon^{-}}[([1-(2-[2-(2-[4-(1 H-p y r r o l-3-y l)-$ butanoylamino]ethoxy)ethoxy]ethoxy)ethyl]-1 $H$-1,2,3-triazol-4-yl)butanoylamino]-L-

lysinmethylester (3b). Reaction time 4 h. Brown oil; yield: $147 \mathrm{mg}(98 \%), \mathrm{R}_{\mathrm{f}} 0.25$ $\left(\mathrm{CH}_{2} \mathrm{Cl}_{2} / \mathrm{MeOH}, 9: 1\right)$.

${ }^{1} \mathrm{H}-\mathrm{NMR}\left(\mathrm{CDCl}_{3}, 500 \mathrm{MHz}\right): \delta 1.47\left(\mathrm{~m}, 4 \mathrm{H}, 2 \mathrm{xCH}_{2}-\mathrm{C}_{2}-\mathrm{CH}_{2}\right), 1.65\left(\mathrm{~m}, 2 \mathrm{H}, \mathrm{CH}-\mathrm{C}_{2}-\mathrm{CH}_{2}\right)$, $1.87\left(\mathrm{~m}, 2 \mathrm{H}, \mathrm{CH}_{2}-\mathrm{C}_{2}-\mathrm{CH}_{2}\right), 1.94$ (m, 2H, CH $\left.2-\underline{\mathrm{C}}_{2}-\mathrm{CO}\right), 2.16$ (t, $2 \mathrm{H}, J$ 7.6, $\left.\mathrm{C}_{\mathrm{ar}}-\underline{\mathrm{C}}_{2}-\mathrm{CH}_{2}\right), 2.20$ (t, $2 \mathrm{H}, J$ 7.1, $\mathrm{CH}_{2}-\underline{\mathrm{C}}_{2}-\mathrm{CO}$ ), 2.47 (t, $2 \mathrm{H}, J$ 7.2, $\mathrm{CH}_{2}-\underline{\mathrm{C}}_{2}-\mathrm{CH}_{2}$ ), 2.69 (t, $2 \mathrm{H}, J$ 7.1, $\mathrm{CH}_{2}-\mathrm{C}_{2}-$

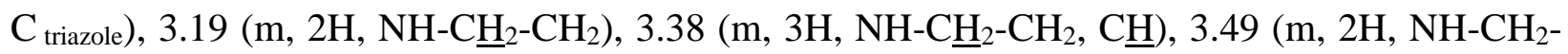
C $\underline{\mathrm{H}}_{2}-\mathrm{O}$ ), 3.53-3.69 (m, 21H, 2xO-C $\left.\underline{\mathrm{H}}_{2}-\underline{\mathrm{C}}_{2}-\mathrm{O}, 2 \mathrm{xC}_{2}-\mathrm{COOCH}_{3}, 3 \mathrm{xOC}_{3}\right), 3.80(\mathrm{t}, 2 \mathrm{H}, J 4.9$, $\left.\mathrm{C}_{2}-\mathrm{CH}_{2}-\mathrm{N}_{\text {triazole }}\right), 4.43$ (t, $2 \mathrm{H}, J$ 4.9, $\left.\mathrm{CH}_{2}-\mathrm{C}_{2}-\mathrm{N}_{\text {triazole }}\right), 5.99$ (m, $\left.1 \mathrm{H}, \underline{\mathrm{C}}_{\mathrm{Py}}\right), 6.19$ (t, 1H, J 3.8, CO-N $\underline{H}), 6.36(\mathrm{t}, 1 \mathrm{H}, J 4.9, \mathrm{CO}-\mathrm{N} \underline{\mathrm{H}}), 6.52\left(\mathrm{~m}, 1 \mathrm{H}, \underline{\mathrm{C}}_{\mathrm{Py}}\right), 6.65\left(\mathrm{~m}, 1 \mathrm{H}, \underline{\mathrm{C}}_{\mathrm{Py}}\right), 7.44(\mathrm{~s}, 1 \mathrm{H}$, $\left.\mathrm{C}_{\text {triazole }}\right), 8.74$ (s, br, $1 \mathrm{H}, \underline{\mathrm{N}}_{\mathrm{Py}}$ ).

${ }^{13} \mathrm{C}-\mathrm{NMR}\left(\mathrm{CDCl}_{3}, 125 \mathrm{MHz}\right): \delta 23.0\left(\mathrm{CH}_{2}-\mathrm{CH}_{2}-\mathrm{CH}_{2}\right), 24.9\left(\mathrm{CH}_{2}-\mathrm{CH}_{2}-\mathrm{C}_{\text {triazole }}\right), 25.7\left(\mathrm{CH}_{2}-\mathrm{CH}_{2}-\right.$ $\left.\mathrm{CH}_{2}\right), 26.4\left(\mathrm{CH}_{2}-\mathrm{CH}_{2}-\mathrm{CH}_{2}\right), 27.1\left(\mathrm{CH}_{2}-\underline{\mathrm{C}} \mathrm{H}_{2}-\mathrm{CO}\right), 28.7\left(\mathrm{CH}_{2}-\underline{\mathrm{CH}}_{2}-\mathrm{CH}_{2}\right), 29.8\left(\mathrm{C}_{\alpha} \mathrm{H}-\underline{\mathrm{CH}}_{2}-\mathrm{CH}_{2}\right)$, $35.6\left(\mathrm{CH}_{2}-\underline{\mathrm{CH}_{2}}-\mathrm{CO}\right), 36.1\left(\mathrm{C}_{\mathrm{ar}}-\underline{\mathrm{CH}}_{2}-\mathrm{CH}_{2}\right), 39.2\left(2 \mathrm{xNH}-\underline{\mathrm{CH}}_{2}-\mathrm{CH}_{2}\right), 50.1\left(\mathrm{CH}_{2}-\underline{\mathrm{CH}}_{2}-\mathrm{N}_{\text {triazole }}\right), 51.5$ $\left(\mathrm{OCH}_{3}\right), 51.7\left(2 \mathrm{xO}^{\mathrm{C}} \mathrm{H}_{3}\right), 52.6\left(2 \mathrm{x}^{-} \mathrm{CH}_{2}-\mathrm{COOCH}_{3}\right), 64.4\left(\underline{\mathrm{C}}_{\alpha} \mathrm{H}\right), 69.5\left(\mathrm{O}-\underline{\mathrm{CH}}_{2}-\mathrm{CH}_{2}-\mathrm{O}\right), 69.9(\mathrm{O}-$ $\left.\underline{\mathrm{CH}}_{2}-\mathrm{CH}_{2}-\mathrm{O}\right), 70.2\left(\mathrm{O}-\mathrm{CH}_{2}-\mathrm{CH}_{2}-\mathrm{O}\right), 70.4\left(\mathrm{O}-\mathrm{CH}_{2}-\mathrm{CH}_{2}-\mathrm{O}\right), 70.5\left(2 \mathrm{xO}-\mathrm{CH}_{2}-\mathrm{CH}_{2}-\mathrm{O}\right), 108.3$ $\left(\underline{\mathrm{CH}}_{\mathrm{Py}}\right), 115.3\left(\underline{\mathrm{CH}}_{\mathrm{Py}}\right), 117.8\left(\underline{\mathrm{CH}}_{\mathrm{Py}}\right), 122.2\left(\underline{\mathrm{CH}}_{\text {triazole }}\right), 122.9\left(\underline{\mathrm{C}}_{\mathrm{q}}, \mathrm{Py}\right), 147.3\left(\underline{\mathrm{C}}_{\mathrm{q}}\right.$, triazole $), 171.9$ $(2 \mathrm{x} \underline{\mathrm{C}}=\mathrm{O}), 172.9(\underline{\mathrm{C}}=\mathrm{O}), 173.1(\underline{\mathrm{C}}=\mathrm{O}), 173.4(\underline{\mathrm{C}}=\mathrm{O})$.

FTIR $\left(\mathrm{cm}^{-1}\right): 3305 v(\mathrm{NH} \&$ triazole $), 2927 v_{\text {as }}\left(\mathrm{CH}_{2}\right), 2865 v_{\mathrm{s}}\left(\mathrm{CH}_{2}\right), 1733 v(\mathrm{C}=\mathrm{O}$ ester $), 1647$ $v(\mathrm{C}=\mathrm{O}), 1537 v\left(\mathrm{C}=\mathrm{C}_{\text {triazole }}\right), 1435 \delta\left(\mathrm{CH}_{2}\right), 1202,1143 v\left(\mathrm{C}-\mathrm{O}_{\text {ester }}\right), 1107 v_{\text {as }}\left(\mathrm{C}-\mathrm{O}-\mathrm{C}_{\mathrm{TEG}}\right), 844$ $\gamma\left(\mathrm{CH}_{\text {triazole }}\right), 729 \delta\left(\mathrm{CH}_{\mathrm{Py}}\right)$.

HRMS (ESI): $m / z$ [M+H] ${ }^{+}$calcd for $\mathrm{C}_{35} \mathrm{H}_{57} \mathrm{~N}_{7} \mathrm{O}_{11}: 752.4189$; found: 752.4187 .

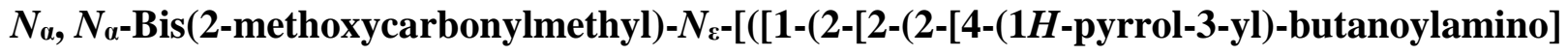
ethoxy)ethoxy]ethoxy)ethyl]-1H-1,2,3-triazol-4-yl)-2-(2-[2-(2-[2-methoxyethoxy]ethoxy) 
ethoxy]ethoxy)acetamido]-L-lysine methyl ester (3c). Reaction time 4 h. Dark brown oil; yield $184 \mathrm{mg}(99 \%), \mathrm{R}_{\mathrm{f}} 0.27\left(\mathrm{CH}_{2} \mathrm{Cl}_{2} / \mathrm{MeOH}, 9: 1\right)$.

${ }^{1} \mathrm{H}-\mathrm{NMR}\left(\mathrm{CDCl}_{3}, 500 \mathrm{MHz}\right): \delta 1.42\left(\mathrm{~m}, 4 \mathrm{H}, 2 \mathrm{xCH}_{2}-\underline{\mathrm{C}}_{2}-\mathrm{CH}_{2}\right), 1.64\left(\mathrm{~m}, 2 \mathrm{H}, \mathrm{CH}-\mathrm{CH}_{2}-\mathrm{CH}_{2}\right)$, $1.83\left(\mathrm{~m}, 2 \mathrm{H}, \mathrm{CH}_{2}-\underline{\mathrm{C}}_{2}-\mathrm{CH}_{2}\right), 2.13$ (t, $2 \mathrm{H}, J$ 7.5, $\left.\mathrm{C}_{\mathrm{ar}}-\mathrm{CH}_{2}-\mathrm{CH}_{2}\right), 2.44$ (t, 2H, J 7.2, $\mathrm{CH}_{2}-\mathrm{C}_{2}-\mathrm{CO}$ ),

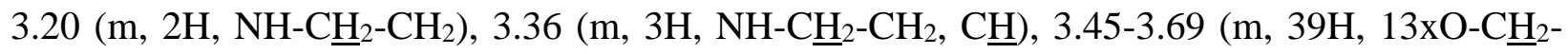
$\mathrm{CH}_{2}-\mathrm{O}, 2 \mathrm{xCH}_{2}-\mathrm{COOCH}_{3}, 3 \mathrm{xOC}_{3}$ ), 3.79 (t, 2H, J 5.0, $\mathrm{C}_{2}-\mathrm{CH}_{2}-\mathrm{N}_{\text {triazole }}$ ), 3.90 (s, 2H, O-C $\underline{H}_{2}-$ $\mathrm{CONH}$ ), 4.44 (t, 2H, J 5.0, $\left.\mathrm{CH}_{2}-\underline{\mathrm{C}}_{2}-\mathrm{N}_{\text {triazole }}\right), 4.59$ (s, 2H, O-C $\left.\underline{\mathrm{H}}_{2}-\mathrm{C}_{\text {triazole }}\right), 5.96$ (m, 1H, $\underline{\mathrm{H}}_{\mathrm{Py}}$ ),

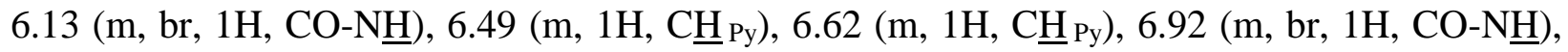
7.65 (s, 1H, $\left.\underline{\mathrm{H}}_{\text {triazole }}\right), 8.73$ (s, br, $\left.1 \mathrm{H}, \underline{\mathrm{N}}_{\mathrm{Py}}\right)$.

${ }^{13} \mathrm{C}-\mathrm{NMR}\left(\mathrm{CDCl}_{3}, 125 \mathrm{MHz}\right): \delta 23.2\left(\mathrm{CH}_{2}-\underline{\mathrm{CH}}_{2}-\mathrm{CH}_{2}\right), 26.3\left(\mathrm{CH}_{2}-\underline{\mathrm{CH}}_{2}-\mathrm{CO}\right), 27.0\left(\mathrm{CH}_{2}-\underline{\mathrm{CH}}_{2}-\right.$ $\left.\mathrm{CH}_{2}\right), 29.2\left(\mathrm{CH}_{2}-\underline{\mathrm{CH}}_{2}-\mathrm{CH}_{2}\right), 30.0\left(\mathrm{C}_{\alpha} \mathrm{H}-\underline{\mathrm{CH}}_{2}-\mathrm{CH}_{2}\right), 36.0\left(\mathrm{C}_{\mathrm{ar}}-\underline{\mathrm{CH}}_{2}-\mathrm{CH}_{2}\right), 38.6\left(\mathrm{NH}-\underline{\mathrm{CH}}_{2}-\mathrm{CH}_{2}\right)$, $39.1\left(\mathrm{NH}-\underline{\mathrm{CH}}_{2}-\mathrm{CH}_{2}\right), 50.1\left(\mathrm{CH}_{2}-\underline{\mathrm{CH}}_{2}-\mathrm{N}_{\text {triazole }}\right), 51.4\left(\mathrm{OC}_{3}\right), 51.6\left(2 \mathrm{xOC} \underline{H}_{3}\right), 52.3\left(2 \mathrm{x}^{-} \mathrm{H}_{2}-\right.$ $\left.\mathrm{COOCH}_{3}\right), 64.5\left(\mathrm{O}-\underline{\mathrm{CH}}_{2}-\mathrm{C}_{\text {triazole }}\right), 64.6(\underline{\mathrm{C}} \alpha \mathrm{H}), 69.3\left(\mathrm{CH}_{2}-\mathrm{CH}_{2}-\mathrm{N}_{\text {triazole }}\right), 69.6\left(\mathrm{O}-\underline{\mathrm{CH}}_{2}-\mathrm{CH}_{2}-\mathrm{O}\right)$, $69.8\left(\mathrm{O}-\underline{\mathrm{CH}}_{2}-\mathrm{CH}_{2}-\mathrm{O}\right), 70.1\left(\mathrm{O}-\underline{\mathrm{CH}}_{2}-\mathrm{CH}_{2}-\mathrm{O}, \mathrm{O}-\underline{\mathrm{CH}}_{2}-\mathrm{CO}\right), 70.3\left(2 \mathrm{xO}-\underline{\mathrm{CH}}_{2}-\mathrm{CH}_{2}-\mathrm{O}\right), 70.5(7 \mathrm{xO}-$ $\left.\underline{\mathrm{CH}}_{2}-\mathrm{CH}_{2}-\mathrm{O}\right), 70.9\left(\mathrm{O}-\underline{\mathrm{CH}}_{2}-\mathrm{CH}_{2}-\mathrm{O}\right), 108.1$ ( $\left.\underline{\mathrm{CH}}_{\mathrm{Py}}\right), 115.2\left(\underline{\mathrm{CH}}_{\mathrm{Py}}\right), 117.7\left(\underline{\mathrm{CH}}_{\mathrm{Py}}\right), 122.8\left(\underline{\mathrm{C}}_{\mathrm{q}}, \mathrm{Py}\right)$, $123.8\left(\underline{\mathrm{CH}}_{\text {triazole }}\right), 144.8\left(\underline{\mathrm{C}}_{\mathrm{q}}\right.$, triazole $), 169.8(\underline{\mathrm{C}}=\mathrm{O}), 171.7(2 \mathrm{x} \underline{\mathrm{C}}=\mathrm{O}), 172.9(\underline{\mathrm{C}}=\mathrm{O}), 173.3(\underline{\mathrm{C}}=\mathrm{O})$.

FTIR $\left(\mathrm{cm}^{-1}\right): 3337 v(\mathrm{NH} \&$ triazole $), 2922 v_{\mathrm{as}}\left(\mathrm{CH}_{2}\right), 2866 v_{\mathrm{s}}\left(\mathrm{CH}_{2}\right), 1732 v\left(\mathrm{C}=\mathrm{O}_{\text {ester }}\right), 1659$ $v(\mathrm{C}=\mathrm{O}), 1535 v\left(\mathrm{C}=\mathrm{C}_{\text {triazole }}\right), 1435 \delta\left(\mathrm{CH}_{2}\right), 1202,1139 v(\mathrm{C}-\mathrm{O}$ ester $), 1099 v_{\text {as }}(\mathrm{C}-\mathrm{O}-\mathrm{C}$ TEG $), 843$ $\gamma\left(\mathrm{CH}_{\text {triazole }}\right), 729 \delta\left(\mathrm{CH}_{\mathrm{Py}}\right)$.

HRMS (ESI): $m / z$ [M+Na] $]^{+}$calcd for $\mathrm{C}_{42} \mathrm{H}_{71} \mathrm{~N}_{7} \mathrm{O}_{16} \mathrm{Na}$ : 952.4850; found: 952.4849. cyclo-(L-Argininyl-glycinyl-L-aspartyl-D-phenylalaninyl- $N_{\gamma}$-[([1-(2-[2-(2-[4-(1H-pyrrol-3yl)-butanoylamino]ethoxy)ethoxy]ethoxy)ethyl]-1H-1,2,3-triazol-4-yl)methylamino]-Lglutamic acid) (3d). Reaction time $5 \mathrm{~h}$. Brown oil; yield: $197 \mathrm{mg}$ (99\%)

${ }^{1} \mathrm{H}-\mathrm{NMR}(\mathrm{DMF}-\mathrm{d} 7,500 \mathrm{MHz}): \delta 1.58\left(\mathrm{~m}, 2 \mathrm{H}, \mathrm{C}_{\alpha} \mathrm{H}-\mathrm{CH}_{2}-\mathrm{C}_{2}, \mathrm{Arg}\right), 1.76\left(\mathrm{~m}, 3 \mathrm{H}, \mathrm{C}_{\alpha} \mathrm{H}-\mathrm{C}_{2}-\right.$ $\left.\mathrm{CH}_{2, \mathrm{Arg}}, \mathrm{CH}_{2}-\underline{\mathrm{C}}_{2}-\mathrm{CH}_{2}\right), 1.83$ (d, $1 \mathrm{H}, J$ 5.1, $\left.\mathrm{C}_{\alpha} \mathrm{H}-\mathrm{C}_{2}-\mathrm{CH}_{2}, \mathrm{Arg}\right), 1.96$ (m, 2H, C ${ }_{\alpha} \mathrm{H}-\mathrm{C}_{2}-\mathrm{CH}_{2}, \mathrm{Glu}$ ), $2.15\left(\mathrm{t}, 4 \mathrm{H}, J\right.$ 7.4, $\left.\mathrm{C}_{\mathrm{ar}}-\underline{\mathrm{C}}_{2}-\mathrm{CH}_{2}, \mathrm{CH}_{2}-\underline{\mathrm{C}}_{2}-\mathrm{CO}_{\mathrm{Glu}}\right), 2.39$ (m, 4H, $\mathrm{CH}_{2}-\underline{\mathrm{C}}_{2}-\mathrm{CO}, \mathrm{C}_{\alpha} \mathrm{H}_{-}-\underline{\mathrm{H}}_{2}-$ $\mathrm{CO}_{\mathrm{Asp}}$ ), 2.83 (s, 1H, $\mathrm{C}_{\alpha} \mathrm{H}-\underline{\mathrm{C}}_{2}-\mathrm{Ph}$ Phe), 3.01 (dd, 1H, $J_{1} 6.8, J_{2} 13.2, \mathrm{C}_{\alpha} \mathrm{H}-\mathrm{C}_{2}-\mathrm{Ph}$ Phe), 3.27 (m, $\left.5 \mathrm{H}, \mathrm{NH}-\underline{\mathrm{H}}_{2}-\mathrm{CH}_{2}-\mathrm{O}, \mathrm{CH}_{2}-\underline{\mathrm{CH}}_{2}-\mathrm{NH}_{\mathrm{Arg}}, 1 \times \underline{\mathrm{C}}_{2}, \mathrm{Gly}\right), 3.42-3.64$ (m, 10H, 5xO-C $\underline{\mathrm{H}}_{2}-\mathrm{CH}_{2}$ ), 3.85 (s, $2 \mathrm{H}, \underline{\mathrm{C}}_{2}-\mathrm{CH}_{2}-\mathrm{N}_{\text {triazole }}$ ), 4.20 (m, $\left.3 \mathrm{H}, \mathrm{C}_{\alpha} \underline{\mathrm{H}}_{\mathrm{Glu}}, \mathrm{C}_{\alpha} \underline{\mathrm{H}}_{\mathrm{Phe}}, 1 \mathrm{xC} \underline{\mathrm{H}}_{2}, \mathrm{Gly}\right), 4.38$ (s, 2H, NH-C$\underline{\mathrm{H}}_{2}-\mathrm{C}_{\text {triazole }}$ ), 4.53 (s, 2H, $\left.\mathrm{CH}_{2}-\underline{\mathrm{C}}_{2}-\mathrm{N}_{\text {triazole }}\right), 4.63$ (d, 1H, J 5.0, $\left.\mathrm{C}_{\alpha} \underline{\mathrm{H}}_{\mathrm{Arg}}\right), 4.79$ (m, 1H, $\mathrm{C}_{\alpha} \underline{\mathrm{H}}_{\text {Asp }}$ ), 5.88 (s, 1H,

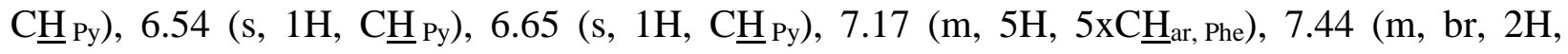

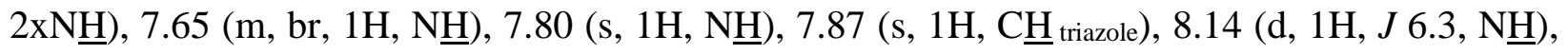

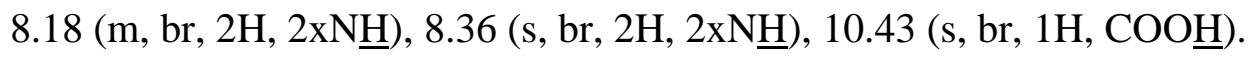

${ }^{13} \mathrm{C}-\mathrm{NMR}(\mathrm{DMF}-\mathrm{d} 7,125 \mathrm{MHz}): \delta 26.1\left(\mathrm{C}_{\alpha} \mathrm{H}-\mathrm{CH}_{2}-\underline{C H}_{2}, \mathrm{Arg}\right), 27.1\left(\mathrm{CH}_{2}-\underline{C H}_{2}-\mathrm{CH}_{2}\right), 28.1\left(\mathrm{CH}_{2}-\right.$

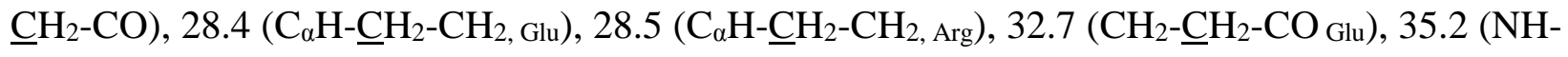
$\left.\underline{\mathrm{CH}_{2}}-\mathrm{C}_{\text {triazole }}\right), 36.1\left(\mathrm{C}_{\alpha} \mathrm{H}-\underline{\mathrm{CH}}_{2}-\mathrm{CO}_{\mathrm{Asp}}, \mathrm{C}_{\mathrm{ar}}-\underline{\mathrm{CH}}_{2}-\mathrm{CH}_{2}\right), 37.8\left(\mathrm{C}_{\alpha} \mathrm{H}-\underline{\mathrm{CH}}_{2}-\mathrm{Ph} \mathrm{Phe}\right), 39.4\left(\mathrm{NH}-\underline{\mathrm{CH}}_{2}-\right.$ $\left.\mathrm{CH}_{2}-\mathrm{O}\right), 41.4\left(\mathrm{CH}_{2}-\underline{\mathrm{CH}_{2}}-\mathrm{NH}_{\mathrm{Arg}}\right), 44.0\left(\underline{\mathrm{CH}}_{2}, \mathrm{Gly}\right), 50.4\left(\mathrm{CH}_{2}-\underline{\mathrm{CH}}_{2}-\mathrm{N}_{\text {triazole }}\right), 53.1\left(\underline{\mathrm{C}}_{a} \mathrm{H}_{\text {Asp }}\right), 54.7$ $\left(\underline{\mathrm{C}}_{\alpha} \mathrm{H}_{\mathrm{Glu}}\right), 55.1\left(\underline{\mathrm{C}}_{\alpha} \mathrm{H} \mathrm{Arg}\right), 55.4\left(\underline{\mathrm{C}}_{\alpha} \mathrm{H} \mathrm{Phe}\right), 69.7\left(\underline{\mathrm{CH}}_{2}-\mathrm{CH}_{2}-\mathrm{N}_{\text {triazole }}\right), 70.2\left(\mathrm{O}-\underline{\mathrm{CH}}_{2}-\mathrm{CH}_{2}-\mathrm{O}\right), 70.3(\mathrm{O}-$ $\left.\underline{\mathrm{CH}}_{2}-\mathrm{CH}_{2}-\mathrm{O}\right), 70.6\left(\mathrm{O}-\underline{\mathrm{CH}}_{2}-\mathrm{CH}_{2}-\mathrm{O}\right), 70.7 \quad\left(\mathrm{O}-\underline{\mathrm{CH}}_{2}-\mathrm{CH}_{2}-\mathrm{O}\right), 70.8 \quad\left(\mathrm{NH}-\mathrm{CH}_{2}-\underline{\mathrm{CH}}_{2}-\mathrm{O}\right), 108.1$ $\left(\underline{\mathrm{CH}}_{\mathrm{Py}}\right), 115.6\left(\underline{\mathrm{CH}}_{\mathrm{Py}}\right), 118.1\left(\underline{\mathrm{CH}}_{\mathrm{Py}}\right), 123.1\left(\underline{\mathrm{C}}_{\mathrm{q}}, \mathrm{Py}\right), 126.7\left(\underline{\mathrm{CH}}_{\text {triazole }}\right), 126.9\left(\underline{\mathrm{CH}}_{\mathrm{ar}}\right), 128.8$ 
(2x $\left.\underline{\mathrm{CH}}_{\mathrm{ar}}\right), 129.9\left(2 \mathrm{x} \underline{\mathrm{CH}}_{\mathrm{ar}}\right), 138.4\left(\underline{\mathrm{C}}_{\mathrm{q}, \text { ar }}\right), 145.9\left(\underline{\mathrm{C}}_{\mathrm{q}}\right.$, triazole $), 158.3\left(\underline{\mathrm{C}}_{\mathrm{q}}=\mathrm{NH}\right), 170.4(\underline{\mathrm{C}}=\mathrm{O}), 171.3$ $(\underline{\mathrm{C}}=\mathrm{O}), 171.9(\underline{\mathrm{C}}=\mathrm{O}), 172.3(\underline{\mathrm{C}}=\mathrm{O}), 172.4(\underline{\mathrm{C}}=\mathrm{O}), 173.1(\underline{\mathrm{C}}=\mathrm{O}), 173.3(\underline{\mathrm{C}}=\mathrm{O}), 173.5(\underline{\mathrm{C}}=\mathrm{O})$.

UPLC-MS: $\quad t_{\mathrm{R}} 2.45 \mathrm{~min} ; \mathrm{m} / \mathrm{z}$ 995.51; (Column UPLC® BEH C18 $2.1 \times 50 \mathrm{~mm}, 1.7 \mu \mathrm{m}$; gradient: acetonitril : water, $05: 95=>50: 05$; flow: $0.6 \mathrm{ml} / \mathrm{min})$.

FTIR $\left(\mathrm{cm}^{-1}\right): 3302 v(\mathrm{NH} \&$ triazole $), 2926 v_{\text {as }}\left(\mathrm{CH}_{2}\right), 2870 v_{\mathrm{s}}\left(\mathrm{CH}_{2}\right), 1652 v(\mathrm{C}=\mathrm{O}), 1541$ $v\left(\mathrm{C}=\mathrm{C}_{\text {triazole }}\right), 1096 v_{\text {as }}\left(\mathrm{C}-\mathrm{O}-\mathrm{C}_{\mathrm{TEG}}\right), 844 \gamma\left(\mathrm{CH}_{\text {triazole }}\right)$.

HRMS (ESI): $m / z$ [M+ H] $]^{+}$calcd for $\mathrm{C}_{45} \mathrm{H}_{67} \mathrm{~N}_{14} \mathrm{O}_{12}$ : 995.5057; found: 995.5058

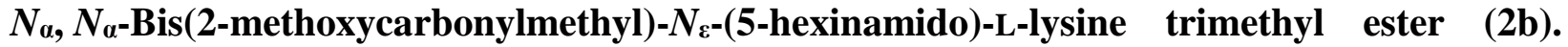
EDC (365 mg, $2.35 \mathrm{mmol})$, 1-hydroxybenzotriazol (HOBt) $(366 \mathrm{mg}, 2.72 \mathrm{mmol}$ ) and DIPEA $(257 \mathrm{mg}, 1.99 \mathrm{mmol})$ were added to a solution of 5-hexynoic acid $\mathbf{4 a}(203 \mathrm{mg}, 1.81 \mathrm{mmol})$ and lysine derivative 5 (605 mg, $1.99 \mathrm{mmol})$ in $\mathrm{CH}_{2} \mathrm{Cl}_{2}$ (18 ml) under argon. After stirring for $20 \mathrm{~h}$ the reaction mixture was diluted with $\mathrm{CH}_{2} \mathrm{Cl}_{2}(18 \mathrm{ml})$ and washed with $3 \mathrm{M}$ aqeous $\mathrm{HCl}(2 \mathrm{x}$ $20 \mathrm{ml})$ and water $(2 \times 25 \mathrm{ml})$. The organic layer was dried over $\mathrm{MgSO}_{4}$ and the solvent was removed under vacuum by a rotary evaporator. The remainder was purified by column chromatography providing $359 \mathrm{mg}(0.90 \mathrm{mmol}, 50 \%)$ of the product $\mathbf{2} \mathbf{b}$ as yellow oil.

$\mathrm{R}_{\mathrm{f}} 0.36\left(\mathrm{CH}_{2} \mathrm{Cl}_{2} / \mathrm{MeOH}, 95: 5\right)$.

${ }^{1} \mathrm{H}-\mathrm{NMR}\left(\mathrm{CDCl}_{3}, 500 \mathrm{MHz}\right): \delta 1.46\left(\mathrm{~m}, 4 \mathrm{H}, \mathrm{C}_{\alpha} \mathrm{H}-\mathrm{C}_{2}-\mathrm{C}_{2}\right), 1.65\left(\mathrm{~m}, 2 \mathrm{H}, \mathrm{CH}_{2}-\mathrm{C}_{2}-\mathrm{CH}_{2}\right), 1.81$ $\left(\mathrm{m}, 2 \mathrm{H}, \mathrm{CH}_{2}-\mathrm{C}_{2}-\mathrm{CH}_{2}\right), 1.93\left(\mathrm{t}, 1 \mathrm{H}, J\right.$ 2.6, $\left.\mathrm{CH}_{2}-\mathrm{C} \equiv \mathrm{C} \underline{\mathrm{H}}\right), 2.20\left(\mathrm{~m}, 2 \mathrm{H}, \mathrm{C}_{2}-\mathrm{C} \equiv \mathrm{CH}\right), 2.82(\mathrm{t}, 2 \mathrm{H}, J$ 7.4, $\mathrm{CH}_{2}-\underline{\mathrm{C}}_{2}-\mathrm{CONH}$ ), 3.20 (m, $\left.2 \mathrm{H}, \mathrm{CH}_{2}-\underline{\mathrm{C}}_{2}-\mathrm{NH}_{2}\right), 3.39$ (t, $1 \mathrm{H}, J$ 7.6, $\mathrm{C}_{a} \underline{\mathrm{H}}$ ), 3.58 (s, 4H, $\left.2 \mathrm{xCH}_{2}-\mathrm{COOCH}_{3}\right), 3.65$ (s, 9H, 3xOCH$\left.\underline{\mathrm{H}}_{3}\right), 6.06$ (s, br, $\left.1 \mathrm{H}, \mathrm{NH}\right)$.

${ }^{13} \mathrm{C}-\mathrm{NMR}\left(\mathrm{CDCl}_{3}, 125 \mathrm{MHz}\right): \delta 18.0\left(\underline{\mathrm{CH}}_{2}-\mathrm{C} \equiv \mathrm{CH}\right), 22.9\left(\mathrm{CH}_{2}-\underline{\mathrm{CH}}_{2}-\mathrm{CH}_{2}\right), 24.4\left(\mathrm{CH}_{2}-\mathrm{CH}_{2}-\mathrm{CH}_{2}\right)$, $28.5\left(\mathrm{CH}_{2}-\mathrm{CH}_{2}-\mathrm{CH}_{2}\right), 29.7\left(\mathrm{C}_{\alpha} \mathrm{H}-\underline{\mathrm{CH}}_{2}-\mathrm{CH}_{2}\right), 35.1\left(\mathrm{CH}_{2}-\mathrm{CH}_{2}-\mathrm{CONH}\right), 39.2\left(\mathrm{CH}_{2}-\mathrm{CH}_{2}-\mathrm{NH}\right), 51.5$ $\left(\mathrm{O}^{-} H_{3}\right), 51.8\left(2 \times \underline{C}_{3}\right), 52.6\left(2 \times \underline{C} H_{2}-\mathrm{COOCH}_{3}\right), 64.3\left(\underline{\mathrm{C}}_{\alpha} \mathrm{H}\right), 69.1\left(\mathrm{CH}_{2}-\mathrm{C} \equiv \underline{\mathrm{CH}}\right), 83.7\left(\mathrm{C}_{\mathrm{q}}, \mathrm{CH}_{2-}\right.$ $\underline{\mathrm{C}} \equiv \mathrm{CH}), 171.9(2 \times \underline{\mathrm{C}}=\mathrm{O}), 172.6(\underline{\mathrm{C}}=\mathrm{O}), 173.2(\underline{\mathrm{C}}=\mathrm{O})$.

FTIR $\left(\mathrm{cm}^{-1}\right): 3293 v(\mathrm{NH} \& \mathrm{CH}: \mathrm{C} \equiv \mathrm{CH}), 2951 v_{\text {as }}\left(\mathrm{CH}_{2}\right), 2865 v_{\mathrm{s}}\left(\mathrm{CH}_{2}\right), 2114 v(\mathrm{C} \equiv \mathrm{C}), 1730$ $v\left(\mathrm{C}=\mathrm{O}_{\text {ester }}\right), 1648 v(\mathrm{C}=\mathrm{O}), 1536 v(\mathrm{~N}-\mathrm{C}=\mathrm{O}), 1434 \delta\left(\mathrm{CH}_{2}\right), 1200,1153 v(\mathrm{C}-\mathrm{O}$ ester $)$. HRMS (ESI): $m / z$ [M+ Na] $]^{+}$calcd for $\mathrm{C}_{19} \mathrm{H}_{30} \mathrm{~N}_{2} \mathrm{O}_{7} \mathrm{Na}$ : 421.1945; found: 421.1945 .

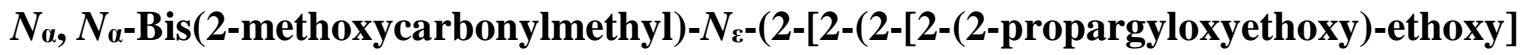
ethoxy)ethoxy]acetamido)-L-lysine trimethyl ester (2c). The glycolic acid derivative $4 \mathbf{b}$ (406 mg, $1.40 \mathrm{mmol}$ ) and the lysine derivative 5 (469 mg, $1.54 \mathrm{mmol}$ ) were reacted in $\mathrm{CH}_{2} \mathrm{Cl}_{2}$ $(14 \mathrm{ml})$ with EDC (283 mg, $1.82 \mathrm{mmol})$, HOBt $(284 \mathrm{mg}, 2.10 \mathrm{mmol})$ and DIPEA (199 mg, $1.54 \mathrm{mmol})$ as shown for the preparation of $2 \mathbf{b}$ resulting in $426 \mathrm{mg}(0.74 \mathrm{mmol}, 53 \%)$ of the product $2 \mathrm{c}$ as yellow oil. $\mathrm{R}_{\mathrm{f}} 0.42\left(\mathrm{CH}_{2} \mathrm{Cl}_{2} / \mathrm{MeOH}, 9: 1\right)$.

${ }^{1} \mathrm{H}-\mathrm{NMR}\left(\mathrm{CDCl}_{3}, 500 \mathrm{MHz}\right): \delta 1.29\left(\mathrm{~m}, 1 \mathrm{H}, \mathrm{C}_{\alpha} \mathrm{H}-\mathrm{C}_{2}-\mathrm{CH}_{2}\right), 1.45\left(\mathrm{~m}, 3 \mathrm{H}, \mathrm{C}_{\alpha} \mathrm{H}-\underline{\mathrm{C}}_{2}-\mathrm{C}_{2}\right), 1.64$ $\left(\mathrm{m}, 2 \mathrm{H}, \mathrm{CH}_{2}-\mathrm{C}_{2}-\mathrm{CH}_{2}\right), 2.40$ (t, $1 \mathrm{H}, J 2.4, \mathrm{CH}_{2}-\mathrm{C} \equiv \mathrm{C} \underline{\mathrm{H}}$ ), 3.21 (dd, $2 \mathrm{H}, J_{1} 7.0, J_{2} 13.4, \mathrm{CH}_{2}-\mathrm{C}_{2}-$ $\left.\mathrm{NH}_{2}\right), 3.35(\mathrm{t}, 1 \mathrm{H}, J$ 7.6, $\mathrm{C} \alpha \underline{\mathrm{H}}), 3.55-3.70\left(\mathrm{~m}, 29 \mathrm{H}, 4 \times \mathrm{XO}-\underline{\mathrm{H}}_{2}-\mathrm{C}_{2}-\mathrm{O}, 2 \mathrm{xC}_{2}-\mathrm{COOCH}_{3}\right.$,

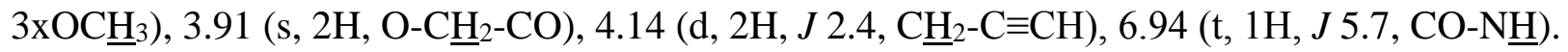

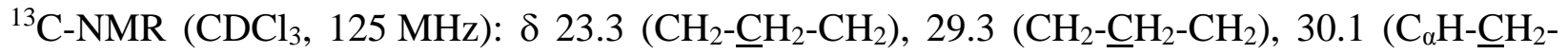
$\left.\mathrm{CH}_{2}\right), 38.6\left(\mathrm{CH}_{2}-\underline{\mathrm{CH}}_{2}-\mathrm{NH}\right), 51.4\left(\mathrm{OCH}_{3}\right), 51.6\left(2 \mathrm{xOCH}_{3}\right), 52.4\left(2 \mathrm{x}^{-} \mathrm{H}_{2}-\mathrm{COOCH}_{3}\right), 58.4\left(\underline{\mathrm{CH}}_{2}-\right.$ $\mathrm{C} \equiv \mathrm{CH}), 64.7\left(\underline{\mathrm{C}}_{a} \mathrm{H}\right), 69.1\left(\mathrm{O}-\underline{\mathrm{CH}}_{2}-\mathrm{CO}\right), 70.2\left(\mathrm{O}-\underline{\mathrm{CH}}_{2}-\mathrm{CH}_{2}-\mathrm{O}\right), 70.4\left(2 \times \mathrm{O}-\underline{\mathrm{CH}}_{2}-\mathrm{CH}_{2}-\mathrm{O}\right), 70.6$ 
(4xO- $\left.\underline{\mathrm{C}} \mathrm{H}_{2}-\mathrm{CH}_{2}-\mathrm{O}\right), 71.0\left(\mathrm{O}-\underline{\mathrm{CH}}_{2}-\mathrm{CH}_{2}-\mathrm{O}\right), 74.6\left(\mathrm{CH}_{2}-\mathrm{C} \equiv \underline{\mathrm{CH}}\right), 79.7 \quad\left(\mathrm{C}_{\mathrm{q}}, \mathrm{CH}_{2}-\underline{\mathrm{C}} \equiv \mathrm{CH}\right), 169.8$ $(\underline{\mathrm{C}}=\mathrm{O}), 171.7(2 \times \underline{\mathrm{C}}=\mathrm{O}), 173.0(\underline{\mathrm{C}}=\mathrm{O})$.

FTIR $\left(\mathrm{cm}^{-1}\right): 3351 \vee(\mathrm{NH}), 3264 \vee(\mathrm{CH}: \mathrm{C} \equiv \mathrm{CH}), 2950 v_{\text {as }}\left(\mathrm{CH}_{2}\right), 2866 v_{\mathrm{s}}\left(\mathrm{CH}_{2}\right), 2113 \vee(\mathrm{C} \equiv \mathrm{C})$, $1731 \vee(\mathrm{C}=\mathrm{O}$ ester $), 1669 \vee(\mathrm{C}=\mathrm{O}), 1531 \vee(\mathrm{N}-\mathrm{C}=\mathrm{O}), 1435 \delta\left(\mathrm{CH}_{2}\right), 1200,1143 \vee(\mathrm{C}-\mathrm{O}$ ester $), 1099$ $v_{\text {as }}\left(\mathrm{C}-\mathrm{O}-\mathrm{C}_{\mathrm{TEG}}\right)$. HRMS (ESI): $\mathrm{m} / z[\mathrm{M}+\mathrm{Na}]^{+}$calcd for $\mathrm{C}_{26} \mathrm{H}_{44} \mathrm{~N}_{2} \mathrm{O}_{12} \mathrm{Na}$ : 599.2786; found: 599.2786.

$\beta$-tert-Butyl-L-aspartyl-D-phenylalaninyl- $N_{\gamma}$-(2-propinylamino)-L-glutamyl- $N_{\omega}-2,2,4,6,7-$ pentamethyl-2,3-dihydrobenzofuran-5-sulfonyl-L-argininyl-glycine $\quad\left(\operatorname{Asp}\left(\mathrm{O}^{t} B u\right)-D-P h e-\right.$ $\left.\mathbf{G l u}\left(\mathbf{C H}_{2}-\mathbf{C} \equiv \mathbf{C H}\right)-\mathbf{A r g}(\mathbf{P b f})-\mathbf{G l y}\right)$ (7). Fmoc-Gly-loaded 2-chlorotrityl resin (2.10 g, $1.14 \mathrm{mmol}$, loading $\left.0.543 \mathrm{mmol}^{*} \mathrm{~g}^{-1}\right)$ was shaken in $N, N$-dimethylformamide (DMF) $(10 \mathrm{ml})$ in a reversed frit filter funnel for $30 \mathrm{~min}$. After removal of the DMF the resin was sequentially washed with $\mathrm{CH}_{2} \mathrm{Cl}_{2} / \mathrm{MeOH} / \mathrm{DIPEA}(17: 2: 1)$ ( $\left.3 \times 6 \mathrm{ml}\right)$, with $\mathrm{CH}_{2} \mathrm{Cl}_{2}$ (3x6 ml), with DMF (3x6 ml) and with $\mathrm{CH}_{2} \mathrm{Cl}_{2}(3 \times 6 \mathrm{ml})$ and then treated with a piperidine/DMF-solution $(1: 4,2 \times 6 \mathrm{ml})$ under shaking for $10 \mathrm{~min}$. The resin was washed with DMF ( $3 \times 6 \mathrm{ml}), \mathrm{CH}_{2} \mathrm{Cl}_{2}(3 \times 6 \mathrm{ml})$ and DMF $(3 \times 6 \mathrm{ml})$ and then combined with a solution of Fmoc-L-Arg(Pbf)-OH (1.48 g, $2.28 \mathrm{mmol}, 2.0$ eq.), HBTU (822 mg, $2.17 \mathrm{mmol}, 1.9$ eq.), HOBt $(310 \mathrm{mg}, 2.28 \mathrm{~mol}, 2.0$ eq.) and $N$-methylmorpholine (NMM) $\left(500 \mu \mathrm{l}, 4.56 \mathrm{mmol}, \rho 0.920 \mathrm{~g}^{*} \mathrm{~cm}^{-3}\right.$, 4.0 eq. $)$ in $\mathrm{CH}_{2} \mathrm{Cl}_{2}(3 \mathrm{ml})$ and DMF (12 ml). After shaking at $\mathrm{rt}$ for $1 \mathrm{~h}$ the solution was removed and the resin washed with DMF ( $3 \times 6 \mathrm{ml}), \mathrm{CH}_{2} \mathrm{Cl}_{2}$ $(3 \times 6 \mathrm{ml})$ and $(3 \times 6 \mathrm{ml})$. For capping the resin was shaken with $30 \%$ acetic anhydride in pyridine $(6 \mathrm{ml})$ for $10 \mathrm{~min}$ and subsequently washed with $30 \%$ acetic anhydride in pyridine $(6 \mathrm{ml})$, DMF ( $3 \times 6 \mathrm{ml}), \mathrm{CH}_{2} \mathrm{Cl}_{2}(3 \times 6 \mathrm{ml})$ and DMF $(3 \times 6 \mathrm{ml})$. Using the same procedures, the following amino acids were coupled in the subsequent steps: Fmoc-L-Glu( $\left.\mathrm{CH}_{2}-\mathrm{C} \equiv \mathrm{CH}\right)-\mathrm{OH}(927 \mathrm{mg}, 2.28 \mathrm{mmol}$, 2.0 eq.), Fmoc-D-Phe-OH ( $884 \mathrm{mg}, 2.28 \mathrm{mmol}, 2.0$ eq.), Fmoc-L-Asp( $\left.\mathrm{O}^{t} \mathrm{Bu}\right)-\mathrm{OH}$ (938 mg, $2.28 \mathrm{mmol}, 2.0$ eq.) affording the Fmoc- $\mathrm{Asp}\left(\mathrm{O}^{t} \mathrm{Bu}\right)-\mathrm{D}-\mathrm{Phe}-\mathrm{Glu}\left(\mathrm{CH}_{2}-\mathrm{C} \equiv \mathrm{CH}\right)-\mathrm{Arg}(\mathrm{Pbf})-\mathrm{Gly}$ sequence at the resin. After deprotection by treatment with piperidine/DMF $(1: 4,2 \times 6 \mathrm{ml})$ and washing with DMF $(3 \times 6 \mathrm{ml}), \mathrm{CH}_{2} \mathrm{Cl}_{2}(3 \times 6 \mathrm{ml})$ and $\mathrm{DMF}(3 \times 6 \mathrm{ml})$ the pentapaeptide was deliberated by shaking with a $1 \%$ solution of TFA in $\mathrm{CH}_{2} \mathrm{Cl}_{2}(4 \times 8 \mathrm{ml})$ for $3 \mathrm{~min}$. The washed solution was poured into a $10 \%$ solution of pyridine in methanol $(40 \mathrm{ml})$, in which also were given all solutions obtained by further washing of the resin with $\mathrm{CH}_{2} \mathrm{Cl}_{2}(3 \times 6 \mathrm{ml})$, methanol ( $3 \times 6 \mathrm{ml}), \mathrm{CH}_{2} \mathrm{Cl}_{2}(3 \times 6 \mathrm{ml})$ and methanol $(3 \times 6 \mathrm{ml})$. All solvents were removed under vacuum by a rotary evaporator. The residue was purified by semi-preparative HPLC providing $885 \mathrm{mg}$ (77\%) of the pentapeptide 7 as a colorless solid. mp $180-182^{\circ} \mathrm{C}$ (partial decomposition).

${ }^{1} \mathrm{H}-\mathrm{NMR}$ (DMF-d $\left.7,300 \mathrm{MHz}\right): \delta 1.39$ (s, 9H, C( $\left.\left(\underline{\mathrm{C}}_{3}\right)_{3}\right), 1.42\left(\mathrm{~d}, 6 \mathrm{H}, \mathrm{C}\left(\mathrm{C}_{3}\right)_{2}\right), 1.57(\mathrm{~m}, 2 \mathrm{H}$,

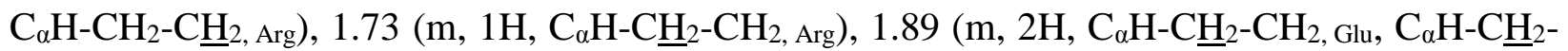

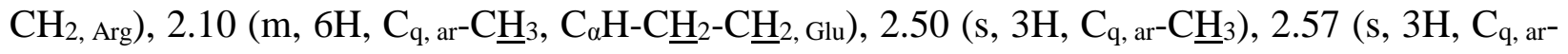
$\left.\mathrm{C}_{3}\right), 2.74$ (m, $2 \mathrm{H}, \mathrm{C}_{\alpha} \mathrm{H}-\underline{\mathrm{C}}_{2}-\mathrm{Ph}$ Phe), 3.00 (m, 4H, $\mathrm{C}_{\alpha} \mathrm{H}-\underline{\mathrm{C}}_{2}-\mathrm{CO}_{\mathrm{Asp}}, \mathrm{CH}_{2}-\mathrm{C} \equiv \mathrm{C} \underline{\mathrm{H}}, \mathrm{C}_{\mathrm{q}}-\mathrm{C}_{2}-\mathrm{C}_{\mathrm{q}}$, ar $)$, $3.16\left(\mathrm{~m}, 3 \mathrm{H}, \mathrm{C}_{\alpha} \mathrm{H}-\mathrm{C}_{2}-\mathrm{CO}_{\mathrm{Asp}}, \mathrm{CH}_{2}-\mathrm{C}_{2}-\mathrm{NH}_{\mathrm{Arg}}\right), 3.63\left(\mathrm{~m}, 1 \mathrm{H}, \mathrm{C}_{2}, \mathrm{Gly}\right), 3.83\left(\mathrm{~m}, 1 \mathrm{H}, \underline{\mathrm{C}}_{2}, \mathrm{Gly}\right)$, $3.95\left(\mathrm{dd}, 2 \mathrm{H}, J_{1} 2.2, J_{2} 5.2, \underline{\mathrm{C}}_{2}-\mathrm{C} \equiv \mathrm{CH}\right), 4.36\left(\mathrm{~m}, 3 \mathrm{H}, \mathrm{C}_{\alpha} \underline{\mathrm{H}}_{\mathrm{Arg}}, \mathrm{C}_{\alpha} \underline{\mathrm{H}} \mathrm{Glu}, \mathrm{C}_{\alpha} \underline{\mathrm{H}} \mathrm{Phe}\right), 4.67(\mathrm{~m}, 1 \mathrm{H}$,

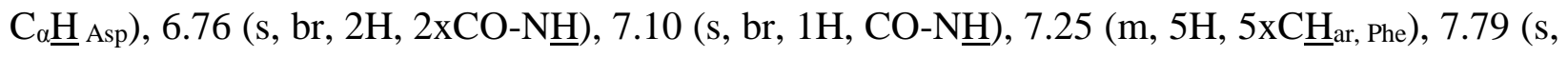




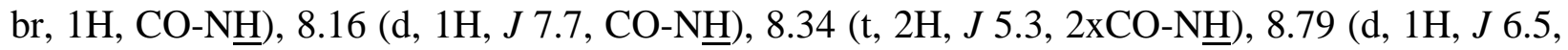
$\mathrm{CO}-\mathrm{N} \underline{\mathrm{H}}), 8.97\left(\mathrm{~s}, \mathrm{br}, 1 \mathrm{H}, \mathrm{C}_{\mathrm{q}}=\mathrm{N} \underline{\mathrm{H}}\right)$.

${ }^{13} \mathrm{C}-\mathrm{NMR}(\mathrm{DMF}-\mathrm{d} 7,75 \mathrm{MHz}): \delta 12.2\left(\mathrm{C}_{\mathrm{q}}, \mathrm{ar}-\underline{\mathrm{C}} \mathrm{H}_{3}\right), 17.9\left(\mathrm{C}_{\mathrm{q}}\right.$, ar- $\left.\underline{\mathrm{CH}}_{3}\right), 19.1\left(\mathrm{C}_{\mathrm{q}}, \mathrm{ar}^{-} \underline{-} \mathrm{H}_{3}\right), 26.0\left(\mathrm{CH}_{2}-\right.$

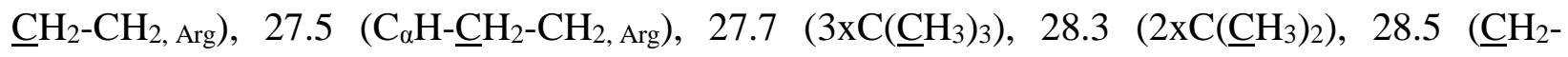
$\mathrm{C} \equiv \mathrm{CH}), 29.3\left(\mathrm{C}_{\alpha} \mathrm{H}-\underline{\mathrm{CH}}_{2}-\mathrm{CH}_{2}, \mathrm{Glu}\right), 32.1\left(\mathrm{CH}_{2}-\underline{\mathrm{CH}}_{2}-\mathrm{CO}_{\mathrm{Glu}}\right), 37.4\left(\mathrm{C}_{\alpha} \mathrm{H}-\underline{\mathrm{CH}}_{2}-\mathrm{Ph}\right), 38.0\left(\mathrm{C}_{\alpha} \mathrm{H}_{-}-\underline{\mathrm{CH}}_{2}-\right.$ $\left.\mathrm{CO}_{\text {Asp }}\right), 40.6\left(\mathrm{CH}_{2}-\underline{\mathrm{CH}}_{2}-\mathrm{NH}_{\mathrm{Arg}}\right), 42.7\left(\underline{\mathrm{CH}}_{2, \mathrm{Gly}}\right), 43.0\left(\mathrm{C}_{\mathrm{q}}-\underline{\mathrm{CH}}_{2}-\mathrm{C}_{\mathrm{q}, \text { ar }}\right), 50.3\left(\underline{\mathrm{C}}_{a} \mathrm{H}_{\mathrm{Arg}}\right), 53.2$ $\left(\underline{\mathrm{C}}_{\alpha} \mathrm{H}_{\mathrm{Phe}}\right), 54.3\left(\underline{\mathrm{C}}_{\alpha} \mathrm{H}_{\mathrm{Glu}}\right), 55.9\left(\underline{\mathrm{C}}_{\alpha} \mathrm{H}_{\mathrm{Asp}}\right), 72.3\left(\mathrm{CH}_{2}-\mathrm{C} \equiv \underline{\mathrm{CH}}\right), 81.1\left(\mathrm{C}_{\mathrm{q}}, \mathrm{CH}_{2}-\underline{\mathrm{C}} \equiv \mathrm{CH}\right), 81.8$ $\left(\underline{\mathrm{C}}\left(\mathrm{CH}_{3}\right)_{3}\right), 86.9\left(\underline{\mathrm{C}}\left(\mathrm{CH}_{3}\right)_{2}\right), 117.1\left(\underline{\mathrm{C}}_{\mathrm{q}, \text { ar }} \mathrm{CH}_{3}\right), 125.1\left(\underline{\mathrm{C}}_{\mathrm{q}}\right.$ ar $\left.\mathrm{CH}_{3}\right), 127.1\left(\underline{\mathrm{C}} \mathrm{H}_{\mathrm{ar}}\right), 129.7\left(2 \underline{\mathrm{CH}}_{\mathrm{ar}}\right)$, $129.7\left(2 \times \underline{C H}_{a r}\right), 132.3\left(\underline{\mathrm{C}}_{\mathrm{q}, \text { ar }}-\mathrm{CH}_{3}\right), 134.7\left(\underline{\mathrm{C}}_{\mathrm{q}, \mathrm{ar}}\right), 137.5\left(\underline{\mathrm{C}}_{\mathrm{q}}\right.$ ar $), 138.2\left(\underline{\mathrm{C}}_{\mathrm{q}, \mathrm{ar}}\right), 157.2\left(\underline{\mathrm{C}}_{\mathrm{q}}=\mathrm{NH}\right)$, $158.3\left(\underline{\mathrm{C}}_{\mathrm{q}}, a_{\mathrm{r}} \mathrm{O}-\mathrm{C}\left(\mathrm{CH}_{3}\right)_{2}-\mathrm{CH}_{2}\right), 164.8(\underline{\mathrm{C}}=\mathrm{O}), 169.8(\underline{\mathrm{C}}=\mathrm{O}), 170.3(\underline{\mathrm{C}}=\mathrm{O}), 172.2(\underline{\mathrm{C}}=\mathrm{O}), 172.4$ $(\underline{\mathrm{C}}=\mathrm{O}), 172.6(2 \times \underline{\mathrm{C}}=\mathrm{O})$.

Semi-preparative HPLC-MS: $\mathrm{t}_{\mathrm{R}} 9.5 \mathrm{~min} ; \mathrm{m} / \mathrm{z}$ 968, (column: Luna-Phenyl-Hexyl 21.2 x $250 \mathrm{~mm}$, $10 \mu \mathrm{m}$; isocratic: methanol : water, $65: 35$;flow: $22.0 \mathrm{ml} / \mathrm{min}$ ).

HRMS (ESI): $m / z$ [M+ H] calcd for $\mathrm{C}_{46} \mathrm{H}_{66} \mathrm{~N}_{9} \mathrm{O}_{12} \mathrm{~S}: 968.4546$; found: 968.4544 .

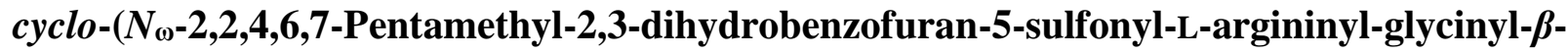
tert-butyl-L-aspartyl-D-phenylalaninyl- $N_{\gamma}$-(2-propinylamino)-L-glutamic acid) cyclo$\left(\mathbf{A r g}(\mathbf{P b f})-\mathbf{G l y}-\mathbf{A s p}\left(\mathbf{O}^{\mathbf{t} B u}\right)-D-P h e-G l u\left(\mathbf{C H}_{2}-\mathbf{C} \equiv \mathbf{C H}\right)\right)(\mathbf{8})$. PyBOP $(169 \mathrm{mg}, 0.325 \mathrm{mmol})$ and NMM $\left(61 \mu 1,0.550 \mathrm{mmol}, \rho 0.92 \mathrm{~g} \mathrm{~cm}^{-3}\right)$ were added to a mixture of the pentapeptide 7 (242 mg, $0.250 \mathrm{mmol})$ and dry DMF (250 ml) under argon. After stirring for $24 \mathrm{~h}$ the solvent was removed under vacuum by a rotary evaporator and the remainder was purified by column chromatography giving rise to the product $8(222 \mathrm{mg}, 93 \%)$ as yellow solid. mp. $215-216^{\circ} \mathrm{C}$ (partial decomposition), $\mathrm{R}_{\mathrm{f}} 0.23\left(\mathrm{CH}_{2} \mathrm{Cl}_{2} / \mathrm{MeOH}, 9: 1\right)$.

${ }^{1} \mathrm{H}-\mathrm{NMR}\left(\mathrm{DMF}-\mathrm{d}_{7}, 300 \mathrm{MHz}\right): \delta 1.37$ (s, 9H, C( $\left.\left(\underline{\mathrm{C}}_{3}\right)_{3}\right), 1.44\left(\mathrm{~s}, 6 \mathrm{H}, \mathrm{C}\left(\mathrm{C}_{3}\right)_{2}\right), 1.72(\mathrm{~m}, 4 \mathrm{H}$, $\mathrm{C}_{\alpha} \mathrm{H}-\mathrm{CH}_{2}-\mathrm{C}_{2}$, Arg, $\left.\mathrm{C}_{\alpha} \mathrm{H}-\mathrm{C}_{2}-\mathrm{CH}_{2}, \mathrm{Glu}\right), 1.91$ (m, 2H, $\left.\mathrm{C}_{\alpha} \mathrm{H}-\mathrm{C}_{2}-\mathrm{CH}_{2}, \mathrm{Arg}\right), 2.06$ (s, 3H, $\mathrm{C}_{\mathrm{q}}$, ar- $\left.\underline{\mathrm{H}}_{3}\right)$, $2.16\left(\mathrm{dd}, 2 \mathrm{H}, J_{1} 8.7, J_{2} 15.7, \mathrm{CH}_{2}-\underline{\mathrm{C}}_{2}-\mathrm{CO}_{\mathrm{Glu}}\right), 2.42\left(\mathrm{dd}, 1 \mathrm{H}, J_{1} 6.0, J_{2} 15.7, \mathrm{C}_{\alpha} \mathrm{H}-\underline{\mathrm{C}}_{2}-\mathrm{CO}_{\mathrm{Asp}}\right)$, $2.52\left(\mathrm{~s}, 3 \mathrm{H}, \mathrm{C}_{\mathrm{q}, ~ a r}-\underline{\mathrm{H}}_{3}\right), 2.59\left(\mathrm{~s}, 3 \mathrm{H}, \mathrm{C}_{\mathrm{q}}\right.$ ar $\left.-\underline{\mathrm{H}}_{3}\right), 2.70\left(\mathrm{~m}, 1 \mathrm{H}, \mathrm{C}_{a} \mathrm{H}-\mathrm{C}_{2}-\mathrm{CO}_{\mathrm{Asp}}\right), 2.90(\mathrm{~m}, 1 \mathrm{H}$, $\mathrm{C}_{\alpha} \mathrm{H}-\underline{\mathrm{C}}_{2}-\mathrm{Ph}$ Phe), 3.01 (m, 2H, $\underline{\mathrm{C}}_{2}$, Gly $), 3.06$ (m, 4H, $\mathrm{C}_{\alpha} \mathrm{H}-\underline{\mathrm{C}}_{2}-\mathrm{Ph}_{\mathrm{Phe}}, \mathrm{CH}_{2}-\mathrm{C} \equiv \mathrm{C} \underline{\mathrm{H}}, \mathrm{C}_{\mathrm{q}}-\mathrm{C}_{2}-$ $\left.\mathrm{C}_{\mathrm{q}, ~ a r}\right), 3.19\left(\mathrm{~m}, 2 \mathrm{H}, \mathrm{CH}_{2}-\mathrm{C}_{2}-\mathrm{NH}_{\mathrm{Arg}}\right), 3.97\left(\mathrm{dd}, 2 \mathrm{H}, J_{1} 2.2, J_{2} 5.7, \mathrm{C}_{2}-\mathrm{C} \equiv \mathrm{CH}\right), 4.21(\mathrm{~m}, 2 \mathrm{H}$,

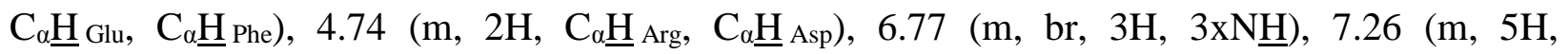

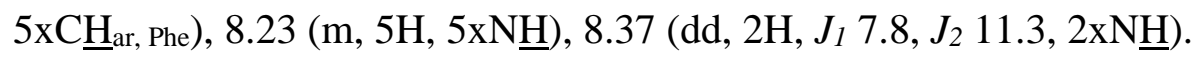

${ }^{13} \mathrm{C}-\mathrm{NMR}(\mathrm{DMF}-\mathrm{d} 7,75 \mathrm{MHz}): \delta 12.4\left(\mathrm{C}_{\mathrm{q}}, \mathrm{ar}-\underline{\mathrm{CH}_{3}}\right), 18.1\left(\mathrm{C}_{\mathrm{q}}\right.$, ar- $\left.\underline{\mathrm{CH}}_{3}\right), 19.3\left(\mathrm{C}_{\mathrm{q}}, \mathrm{ar}-\underline{\mathrm{C}} \mathrm{H}_{3}\right), 26.6\left(\mathrm{C}_{\alpha} \mathrm{H}-\right.$

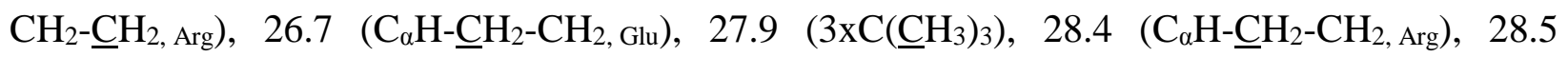
$\left(2 \times C\left(\underline{\mathrm{C}} \mathrm{H}_{3}\right)_{2}\right), 28.7\left(\underline{\mathrm{CH}}_{2}-\mathrm{C} \equiv \mathrm{CH}\right), 32.5\left(\mathrm{CH}_{2}-\underline{\mathrm{CH}}_{2}-\mathrm{CO}_{\mathrm{Glu}}\right), 37.2\left(\mathrm{C}_{\alpha} \mathrm{H}-\underline{\mathrm{CH}}_{2}-\mathrm{CO}_{\mathrm{Asp}}\right), 37.8\left(\mathrm{C}_{\alpha} \mathrm{H}-\right.$ $\underline{\mathrm{CH}}_{2}-\mathrm{Ph}$ Phe $), 40.9\left(\mathrm{CH}_{2}-\underline{\mathrm{CH}}_{2}-\mathrm{NH}_{\mathrm{Arg}}\right), 43.3\left(\underline{\mathrm{CH}}_{2, \mathrm{Gly}}\right), 46.6\left(\mathrm{C}_{\mathrm{q}}-\underline{\mathrm{CH}}_{2}-\mathrm{C}_{\mathrm{q}, \text { ar }}\right), 50.1\left(\underline{\mathrm{C}}_{\alpha} \mathrm{H}_{\mathrm{Asp}}\right), 53.3$ $\left(\underline{\mathrm{C}}_{\alpha} \mathrm{H}_{\mathrm{Glu}}\right), 55.2\left(\underline{\mathrm{C}}_{\alpha} \mathrm{H}_{\mathrm{Arg}}\right), 55.3\left(\underline{\mathrm{C}}_{\alpha} \mathrm{H} \mathrm{Phe}\right), 72.5\left(\mathrm{CH}_{2}-\mathrm{C} \equiv \underline{\mathrm{CH}}\right), 80.6\left(\mathrm{C}_{\mathrm{q}}, \mathrm{CH}_{2}-\underline{\mathrm{C}} \equiv \mathrm{CH}\right), 81.6$ $\left(\underline{\mathrm{C}}\left(\mathrm{CH}_{3}\right)_{3}\right), 87.0\left(\underline{\mathrm{C}}\left(\mathrm{CH}_{3}\right)_{2}\right), 117.2\left(\underline{\mathrm{C}}_{\mathrm{q}, \mathrm{ar}}-\mathrm{CH}_{3}\right), 125.2\left(\underline{\mathrm{C}}_{\mathrm{q}}\right.$ ar $\left.-\mathrm{CH}_{3}\right), 127.0\left(\underline{\mathrm{CH}_{a r}}\right), 128.8\left(2 \underline{\mathrm{C}}_{\mathrm{ar}}\right)$, $129.9\left(2 \times \underline{C}_{\text {ar }}\right), 132.5\left(\underline{\mathrm{C}}_{\mathrm{q}, \text { ar }}-\mathrm{CH}_{3}\right), 135.3\left(\underline{\mathrm{C}}_{\mathrm{q}, \mathrm{ar}}\right), 138.3\left(\underline{\mathrm{C}}_{\mathrm{q}, \text { ar }}\right), 138.4\left(\underline{\mathrm{C}}_{\mathrm{q}, \text { ar }}\right), 157.3\left(\underline{\mathrm{C}}_{\mathrm{q}, \text { ar }} \mathrm{O}-\mathrm{C}\right.$ $\left.\mathrm{C}\left(\mathrm{CH}_{3}\right)_{2}-\mathrm{CH}_{2}\right), 158.5\left(\underline{\mathrm{C}}_{\mathrm{q}}=\mathrm{NH}\right), 170.1(\underline{\mathrm{C}}=\mathrm{O}), 170.3(\underline{\mathrm{C}}=\mathrm{O}), 171.3(\underline{\mathrm{C}}=\mathrm{O}), 171.9(\underline{\mathrm{C}}=\mathrm{O}), 172.0$ $(\underline{\mathrm{C}}=\mathrm{O}), 172.4(\underline{\mathrm{C}}=\mathrm{O}), 172.7(\underline{\mathrm{C}}=\mathrm{O})$.

HRMS (ESI): $m / z$ [M+ H] $]^{+}$calcd for $\mathrm{C}_{46} \mathrm{H}_{64} \mathrm{~N}_{9} \mathrm{O}_{11} \mathrm{~S}: 950.4446$; found: 950.4452 . 
cyclo-(L-Argininyl-glycinyl-L-aspartyl-D-phenylalaninyl- $N_{\gamma}$-(2-propinylamino)-L-glutamic acid) cyclo-(Arg-Gly-Asp-D-Phe-Glu(CH2-C $\equiv \mathbf{C H})$ ) (2d). The cyclic pentapeptide 8 (342 mg, $0.360 \mathrm{mmol}$ ) was combined with a solution of TFA/triethylsilane/water (95:2.5:2.5) (6 ml) under stirring and ice cooling. The mixture was stirred under ice cooling for $30 \mathrm{~min}$ and then at $\mathrm{rt}$ for 2 h. $\mathrm{CH}_{2} \mathrm{Cl}_{2}(20 \mathrm{ml})$ was added and the solvents were distilled off under vacuum by a rotary evaporator. The remainder was dissolved in TFA $(2 \mathrm{ml})$, combined with ice-cooled diethyl ether $(8 \mathrm{ml})$ to precipitate the product, centrifuged, and the supernatant was removed by decantation. This treatment was repeated twice. The colorless solid product was dried by lyophilization providing the cyclic peptide $\mathbf{2 d}\left(203 \mathrm{mg}, 88 \%\right.$ ) as colorless solid. mp 219-221 ${ }^{\circ} \mathrm{C}$ (partial decomposition).

${ }^{1} \mathrm{H}-\mathrm{NMR}\left(\mathrm{DMF}-\mathrm{d}_{7}, 500 \mathrm{MHz}\right): \delta 1.61\left(\mathrm{~m}, 3 \mathrm{H}, \mathrm{C}_{\alpha} \mathrm{H}-\mathrm{C}_{2}-\underline{\mathrm{C}}_{2}, \mathrm{Arg}, \mathrm{C}_{\alpha} \mathrm{H}-\mathrm{C}_{2}-\mathrm{CH}_{2}, \mathrm{Glu}\right), 1.85$ (m, $\left.1 \mathrm{H}, \mathrm{C}_{\alpha} \mathrm{H}-\underline{\mathrm{C}}_{2}-\mathrm{CH}_{2}, \mathrm{Arg}\right), 1.98\left(\mathrm{~m}, 2 \mathrm{H}, \mathrm{C}_{\alpha} \mathrm{H}-\mathrm{C}_{2}-\mathrm{CH}_{2}, \mathrm{Glu}\right), 2.15$ (m, 2H, $\left.\mathrm{CH}_{2}-\underline{\mathrm{C}}_{2}-\mathrm{CO}_{\mathrm{Glu}}\right), 2.48$ (dd, $1 \mathrm{H}, J_{1} 4.6, J_{2} 16.2, \mathrm{C}_{\alpha} \mathrm{H}-\underline{\mathrm{C}}_{2}-\mathrm{CO}_{\mathrm{Asp}}$ ), 2.88 (m, 2H, $\mathrm{C}_{\alpha} \mathrm{H}-\underline{\mathrm{C}}_{2}-\mathrm{CO}_{\mathrm{Asp}}, \mathrm{C}_{\alpha} \mathrm{H}-\mathrm{C}_{2}-\mathrm{Ph} \mathrm{Phe}$ ), 3.06 (m, $2 \mathrm{H}, \mathrm{C}_{\alpha} \mathrm{H}-\underline{\mathrm{CH}}_{2}-\mathrm{Ph}$ Phe $\mathrm{CH}_{2}-\mathrm{C} \equiv \mathrm{C} \underline{\mathrm{H}}$ ), 3.35 (m, 3H, $\left.\mathrm{CH}_{2}-\mathrm{C}_{2}-\mathrm{NH}_{\mathrm{Arg}}, 1 \times \underline{\mathrm{CH}}_{2}, \mathrm{Gly}\right), 3.96$ (s, 2H, $\left.\mathrm{C}_{2}-\mathrm{C} \equiv \mathrm{CH}\right), 4.23$ (m, 3H, $\left.\mathrm{C}_{\alpha} \underline{\mathrm{H}}_{\mathrm{Glu}}, \mathrm{C}_{\alpha} \underline{\mathrm{H}}_{\mathrm{Phe}}, 1 \mathrm{xC} \underline{\mathrm{H}}_{2}, \mathrm{Gly}\right), 4.67$ (dd, 1H, $J_{1} 6.5, J_{2} 13.5, \mathrm{C}_{\alpha} \underline{\mathrm{H}}_{\mathrm{Arg}}$ ),

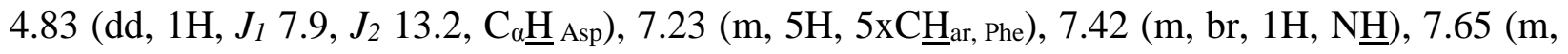

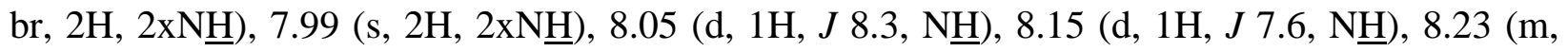
$2 \mathrm{H}, 2 \mathrm{xN} \underline{\mathrm{H}}), 8.35(\mathrm{~m}, 2 \mathrm{H}, \mathrm{N} \underline{\mathrm{H}}, \mathrm{COO} \underline{\mathrm{H}})$.

${ }^{13} \mathrm{C}-\mathrm{NMR}\left(\mathrm{DMF}-\mathrm{d}_{7}, 125 \mathrm{MHz}\right): \delta 26.0\left(\mathrm{C}_{\alpha} \mathrm{H}-\mathrm{CH}_{2}-\underline{\mathrm{CH}}_{2, \mathrm{Arg}}\right), 28.3\left(\mathrm{C}_{\alpha} \mathrm{H}-\underline{\mathrm{CH}}_{2}-\mathrm{CH}_{2}, \mathrm{Glu}\right), 28.5\left(\mathrm{C}_{\alpha} \mathrm{H}-\right.$ $\left.\underline{\mathrm{CH}}_{2}-\mathrm{CH}_{2}, \mathrm{Arg}\right), 28.6\left(\underline{\mathrm{CH}}_{2}-\mathrm{C} \equiv \mathrm{CH}\right), 32.5\left(\mathrm{CH}_{2}-\underline{\mathrm{CH}}_{2}-\mathrm{CO}{ }_{\mathrm{Glu}}\right), 36.6\left(\mathrm{C}_{\alpha} \mathrm{H}-\underline{\mathrm{CH}}_{2}-\mathrm{CO}_{\mathrm{Asp}}\right), 37.9\left(\mathrm{C}_{\alpha} \mathrm{H}-\right.$ $\left.\underline{\mathrm{CH}}_{2}-\mathrm{Ph}{ }_{\mathrm{Phe}}\right), 41.4 \quad\left(\mathrm{CH}_{2}-\underline{\mathrm{CH}}_{2}-\mathrm{NH}_{\mathrm{Arg}}\right), 44.0 \quad\left(\underline{\mathrm{CH}}_{2, \mathrm{Gly}}\right), 50.0 \quad\left(\underline{\mathrm{C}}_{\alpha} \mathrm{H}_{\mathrm{Asp}}\right), 53.0 \quad\left(\underline{\mathrm{C}}_{\alpha} \mathrm{H}_{\mathrm{Glu}}\right), 55.2$ $\left(\underline{\mathrm{C}}_{\alpha} \mathrm{H}_{\mathrm{Arg}}\right), 55.4\left(\underline{\mathrm{C}}_{\alpha} \mathrm{H}_{\mathrm{Phe}}\right), 72.5 \quad\left(\mathrm{CH}_{2}-\mathrm{C} \equiv \underline{\mathrm{CH}}\right), 81.5 \quad\left(\mathrm{C}_{\mathrm{q}}, \mathrm{CH}_{2}-\underline{\mathrm{C}} \equiv \mathrm{CH}\right), 126.9\left(\underline{\mathrm{CH}}_{\mathrm{ar}}\right), 128.8$ $\left(2 \times \underline{C H}_{\mathrm{ar}}\right), 129.9\left(2 \mathrm{x} \underline{\mathrm{CH}}_{\mathrm{ar}}\right), 138.4\left(\underline{\mathrm{C}}_{\mathrm{q}, ~ a r}\right), 158.2\left(\underline{\mathrm{C}}_{\mathrm{q}}=\mathrm{NH}\right), 170.3(\underline{\mathrm{C}}=\mathrm{O}), 171.3(\underline{\mathrm{C}}=\mathrm{O}), 171.9$ $(\underline{\mathrm{C}}=\mathrm{O}), 172.1(\underline{\mathrm{C}}=\mathrm{O}), 172.3(\underline{\mathrm{C}}=\mathrm{O}), 172.5(\underline{\mathrm{C}}=\mathrm{O}), 173.0(\underline{\mathrm{C}}=\mathrm{O})$.

HRMS (ESI): $m / z$ [M+ H] $]^{+}$calcd for $\mathrm{C}_{29} \mathrm{H}_{40} \mathrm{~N}_{9} \mathrm{O}_{8}$ : 642.3000; found: 642.3002

\section{Acknowledgements}

Financial support by Romanian-EU program POS-CCE, Axa II, project nr. 550/2010 is gratefully acknowledged. We thank Dr. Joachim Leistner for HPLC and UPLC investigations.

\section{References}

1. Rodriguez, J.; Grande, H.-J.; Otero, T. F., in Handbook of Organic Conductive Molecules and Polymers; Nalva, H. S., Ed., John Wiley \& Sons, New York, 1997, p. 453

2. Wang, L. X.; Li, X. G.; Yang, Y. L. React. Funct. Polym. 2001, 47, 125.

3. Razola, S. S.; Ruiz, B. L.; Diez, N. M.; Mark, H. B.; Kauffmann, J. M. Biosens. Bioelectron. 2002, 17, 921.

4. Fan, L. Z.; Maier, J. Electrochem. Commun. 2006, 8, 937. 
5. Bose, S.; Kim, N. H.; Kuila, T.; Lau, K. T.; Lee, J. H. Nanotechnology 2011, 22, 295202

6. Nan, A.; Karsten, S.; Craciunescu, I.; Turcu, R.; Vekas, L.; Liebscher, J. Arkivoc 2008, (xv), 307.

7. Turcu, R.; Pana, O.; Nan, A.; Craciunescu, I.; Chauvet, O.; Payen, C. J. Phys. D: Appl. Phys. 2008, 41, 245002.

8. Turcu, R.; Nan, A.; Craciunescu, I.; Liebscher, J.; Pana, O.; Bica, D.; Vekas, L.; Mijangos, C. J. Optoelectr. Adv. Mater. 2008, 10, 2237.

9. Karsten, S.; Ameen, M. A.; Kalläne, S. I.; Nan, A.; Turcu, R.; Liebscher, J. Synthesis 2010, 3021.

10. Nan, A.; Turcu, R.; Bratu, I.; Leostean, C.; Chauvet, O.; Gautron, E.; Liebscher, J. Arkivoc 2010, $(x), 185$.

11. Wuang, S. C.; Neoh, K. G.; Kang, E. T.; Pack, D. W.; Leckband, D. E. Biomaterials 2008, $29,2270$.

12. Wuang, S. C.; Neoh, K. G.; Kang, E. T.; Pack, D. W.; Leckband, D. E. Macromol. Rapid Commun. 2007, 28, 816.

13. Lu, A. H.; Salabas, E. L.; Schuth, F. Angew. Chem. Int. Ed. 2007, 46, 1222.

14. Hildebrandt, N.; Hermsdorf, D.; Signorell, R.; Schmitz, S. A.; Diederichsen, U. Arkivoc 2007, (v), 79.

15. Nan, A.; Craciunescu, I.; Turcu, R. In Aspects on Fundaments and Applications of Conducting Polymers; de Jesus Motheo, A., Ed.; InTech: Rijeka, 2011, p 159.

16. KorriYoussoufi, H.; Garnier, F.; Srivastava, P.; Godillot, P.; Yassar, A. J. Am. Chem. Soc. 1997, 119, 7388.

17. Rodrigez, L. M. T.; Billon, M.; Roget, A.; Bidan, G. J. Electroanal. Chem. 2002, 523, 70.

18. Meldal, M.; Tornoe, C. W. Chem. Rev. 2008, 108, 2952.

19. Tornoe, C. W.; Christensen, C.; Meldal, M. J. Org. Chem. 2002, 67, 3057.

20. Rostovtsev, V. V.; Green, L. G.; Fokin, V. V.; Sharpless, K. B. Angew. Chem. Int. Ed. 2002, $41,2596$.

21. Kalläne, S. I.; Diploma Thesis, Humboldt-University Berlin, 2010.

22. Aleman, C.; Casanovas, J.; Torras, J.; Bertran, O.; Armelin, E.; Oliver, R.; Estrany, F. Polymer 2008, 49, 1066.

23. Garnier, F.; Korri-Youssoufi, H.; Srivastava, P.; Mandrand, B.; Delair, T. Synth. Met. 1999, $100,89$.

24. Chindarkar, N. S.; Franz, A. H. Arkivoc 2008, (xv) 21.

25. Susumu, K.; Uyeda, H. T.; Medintz, I. L.; Pons, T.; Delehanty, J. B.; Mattoussi, H. J. Am. Chem. Soc. 2007, 129, 13987.

26. Hussein, W. M.; Ross, B. P.; Landsberg, M. J.; Levy, D.; Hankamer, B.; McGeary, R. P. J. Org. Chem. 2009, 74, 1473.

27. Gurrath, M.; Müller, G.; Kessler, H.; Aumailley, M.; Timpl, R. Eur. J. Biochem. 1992, 210, 911.

28. Paleček, J.; Draeger, G.; Kirschning, A. Synthesis 2011, 653. 
29. Haubner, R.; Gratias, R.; Diefenbach, B.; Goodman, S. L.; Jonczyk, A.; Kessler, H. J. Am. Chem. Soc. 1996, 118, 7461.

30. Dijkgraaf, I.; Rijnders, A. Y.; Soede, A.; Dechesne, A. C.; van Esse, G. W.; Brouwer, A. J.; Corstens, F. H. M.; Boerman, O. C.; Rijkers, D. T. S.; Liskamp, R. M. J. Org. Biomol. Chem. 2007, 5, 935.

31. Boturyn, D.; Dumy, P. Tetrahedron Lett. 2001, 42, 2787.

32. Tran, V. M.; Victor, X. V.; Yockman, J. W.; Kuberan, B. Glycoconjugate J. 2010, 27, 625.

33. Karsten, S.; Nan, A.; Turcu, R.; Liebscher, J. J. Polym. Sci. A. 2012, 50, 3986. 